\title{
Inverse Problems for a Parabolic Integrodifferential Equation in a Convolutional Weak Form
}

\author{
Kairi Kasemets and Jaan Janno \\ Department of Mathematics, Tallinn University of Technology, Ehitajate tee 5, 19086 Tallinn, Estonia \\ Correspondence should be addressed to Jaan Janno; janno@ioc.ee
}

Received 4 October 2012; Accepted 23 November 2012

Academic Editor: Maurizio Grasselli

Copyright ( $\odot 2013$ K. Kasemets and J. Janno. This is an open access article distributed under the Creative Commons Attribution License, which permits unrestricted use, distribution, and reproduction in any medium, provided the original work is properly cited.

We deduce formulas for the Fréchet derivatives of cost functionals of several inverse problems for a parabolic integrodifferential equation in a weak formulation. The method consists in the application of an integrated convolutional form of the weak problem and all computations are implemented in regular Sobolev spaces.

\section{Introduction}

Many methods to solve inverse problems (e.g., the Landweber iteration, conjugate gradient method) use the Fréchet derivatives of the cost functionals of these problems [1]. The explicit formula for the Fréchet derivative in terms of the variation of the unknowns of the inverse problem contains the solution of an adjoint problem.

The derivation of the explicit formula for such a Fréchet derivative includes testing the direct problem with the solution of the adjoint problem and vice versa: testing the adjoint problem with the solution of the direct problem. In the case of the parabolic weak problem, such a procedure is cumbersome, because of the asymmetry of the properties of the solution and the test function. In the classical formulation of the parabolic weak problem (see, e.g., [2] and also (19) below), the test function must have higher time regularity than the weak solution. This means that in case of nonsmooth coefficients neither the solution of the direct problem nor the solution of the adjoint problem can be used as a test function. Another formulation of the weak parabolic problem consists in reducing the problem to an abstract Cauchy problem over the time variable (see, e.g., [3]). In such a case, a partial integration over the time has to be implemented within singular distributions in the derivation procedure.

In this paper, we present a new method that enables the deduction of the formulas for the Fréchet derivatives for cost functionals of inverse problems related to weak solutions of parabolic problems. The method is based on an integrated convolutional form of the weak direct problem. The requirements to the test function are weaker than in the classical case and coincide with the properties of the solution of the direct problem. All computations in the deduction procedure can be implemented within usual regular Sobolev spaces.

More precisely, we will consider inverse problems related to a parabolic integrodifferential equation that occur in heat flow with memory [4-6]. This equation contains a time convolution. Therefore, the convolutional form of the weak problem is especially suitable. Supposedly, the proposed method can be generalised to parabolic systems, as well.

\section{Formal Direct Problem: Notation}

Let $\Omega$ be an $n$-dimensional domain, where $n \geq 1$, and $\Gamma$ be the boundary of $\Omega$. Let $\Gamma=\Gamma_{1} \cup \Gamma_{2}$ where either $\Gamma_{1}$ or $\Gamma_{2}$ is allowed to be an empty set. In case $n \geq 2$, we assume that $\Gamma$ is sufficiently smooth, meas $\Gamma_{1} \cap \Gamma_{2}=0$, and for any $j \in\{1 ; 2\}$ it holds either $\Gamma_{j}=\emptyset$ or meas $\Gamma_{j}>0$. Denote

$$
\Omega_{t}=\Omega \times(0, t), \quad \Gamma_{1, t}=\Gamma_{1} \times(0, t), \quad \Gamma_{2, t}=\Gamma_{2} \times(0, t),
$$


for $t \geq 0$. Consider the problem (direct problem) to find $u(x, t): \Omega_{T} \rightarrow \mathbb{R}$ such that

$$
\begin{gathered}
u_{t}=A u-m * A u+f+\nabla \cdot \phi \quad \text { in } \Omega_{T}, \\
u=u_{0} \quad \text { in } \Omega \times\{0\}, \\
u=g \quad \text { in } \Gamma_{1, T}, \\
-v_{A} \cdot \nabla u+m * v_{A} \cdot \nabla u=9 u+h+v \cdot \phi \quad \text { in } \Gamma_{2, T},
\end{gathered}
$$

where $T>0$ is a fixed number,

$$
\begin{gathered}
A v=\sum_{i, j=1}^{n}\left(a_{i j} v_{x_{j}}\right)_{x_{i}}+a v, \\
v_{A}=\left(\left.\sum_{j=1}^{n} a_{i j} v_{j}\right|_{i=1, \ldots, n}\right), \\
v=\left(v_{1}, \ldots, v_{n}\right)-\text { outer normal of } \Gamma_{2},
\end{gathered}
$$

$a_{i j}, a, u_{0}: \Omega \rightarrow \mathbb{R}, f: \Omega_{T} \rightarrow \mathbb{R}, \phi: \Omega_{T} \rightarrow \mathbb{R}^{n}, g:$ $\Gamma_{1, T} \rightarrow \mathbb{R}, \vartheta: \Gamma_{2} \rightarrow \mathbb{R}, h: \Gamma_{2, T} \rightarrow \mathbb{R}, m:(0, T) \rightarrow \mathbb{R}$ are given functions, the subscripts $t, x_{j}, x_{i}$ denote the partial derivatives and

$$
m * w(t)=\int_{0}^{t} m(t-\tau) w(\tau) d \tau
$$

denotes the time convolution. In case $\Gamma_{1}=\emptyset\left(\Gamma_{2}=\emptyset\right)$, the boundary condition (4) and (5) is dropped.

The problem (2)-(5) describes the heat flow in the body $\Omega$ with the thermal memory. Concerning the physical background, we refer the reader to $[4,6,7]$. The solution $u$ is the temperature of the body and $m$ is the heat flux relaxation (or memory) kernel. The boundary condition (5) is of the third kind where the term $-v_{A} \cdot \nabla u+m * v_{A} \cdot \nabla u$ equals the heat flux in the direction of the conormal vector.

Let us introduce some additional notations. Let $t>0$. We use the Sobolev spaces

$$
\begin{aligned}
W_{2}^{l}(\Omega)=\{v & : \Omega \longrightarrow \mathbb{R}:\|v\|_{W_{2}^{l}(\Omega)} \\
& \left.:=\left[\sum_{|\alpha| \leq l}\left\|D_{x}^{\alpha} v\right\|_{L^{2}(\Omega)}^{2}\right]^{1 / 2}<\infty\right\} .
\end{aligned}
$$

Here, $l=0,1,2, \ldots, \alpha=\left(\alpha_{1}, \ldots, \alpha_{n}\right)$ is the multiindex, $|\alpha|=$ $\alpha_{1}+\cdots+\alpha_{n}$ and $D_{x}^{\alpha} v=\partial^{|\alpha|} v / \partial x_{1}^{\alpha_{1}} \cdots \partial x_{n}^{\alpha_{n}}$. Further, let $X$ be a Banach space. We denote by $C([0, t] ; X)$ the space of abstract continuous functions from $[0, t]$ to $X$ endowed with the usual maximum norm $\|v\|_{C([0, t] ; X)}:=\max _{s \in[0, t]}\|v(s)\|$. Moreover, let

$$
\begin{array}{r}
L^{p}((0, t) ; X):=\left\{v:(0, t) \longrightarrow X:\|v\|_{L^{p}((0, t) ; X)}\right. \\
\left.\geq\left[\int_{0}^{t}\|v(s)\|^{p} d s\right]^{1 / p}<\infty\right\}
\end{array}
$$

$$
\text { for } 1<p<\infty \text {, }
$$

$$
\begin{array}{r}
L^{\infty}((0, t) ; X):=\left\{v:(0, t) \longrightarrow X:\|v\|_{L^{\infty}((0, t) ; X)}\right. \\
\left.=\text { ess } \sup _{t \in(0, T)}\|v(s)\|<\infty\right\} .
\end{array}
$$

By means of these spaces, we define the following important functional spaces:

$$
\begin{aligned}
& \mathcal{U}\left(\Omega_{t}\right)=C\left([0, t] ; L^{2}(\Omega)\right) \cap L^{2}\left((0, t) ; W_{2}^{1}(\Omega)\right), \\
& \mathcal{U}_{0}\left(\Omega_{t}\right)=\left\{\eta \in \mathcal{U}\left(\Omega_{t}\right):\left.\eta\right|_{\Gamma_{1, t}}=0 \text { in case } \Gamma_{1} \neq \emptyset\right\} .
\end{aligned}
$$

Convention. In case $n=1$, the integrals $\int_{\Gamma_{j}} v(x) d \Gamma, j=1,2$ are equal to $\sum_{k=1}^{K} v\left(x_{k}\right)$, where $x_{k} \in \Gamma_{j}$ and $K$ is the number of points in $\Gamma_{j}$, and $L_{p}\left(\Gamma_{j}\right)$ is simply $\mathbb{R}^{K}$.

\section{Weak Direct Problem and Its Convolutional Form}

Let us return to the direct problem (2)-(5). Throughout the paper we assume the following basic regularity conditions on the coefficients, the kernel, and the initial and boundary functions:

$$
\begin{gathered}
a_{i j} \in L^{\infty}(\Omega), \quad a_{i j}=a_{j i}, \quad \vartheta \in C\left(\bar{\Gamma}_{2}\right), \quad \vartheta \geq 0 \\
a \in L^{q_{1}}(\Omega), \quad \text { where } q_{1}=1 \text { if } n=1, \quad q_{1}>\frac{n}{2} \text { if } n \geq 2 \\
m \in L^{1}(0, T), \quad g \in L^{2}\left((0, T) ; W_{2}^{1}(\Omega)\right), \\
g_{t} \in L^{2}\left(\Omega_{T}\right), \\
q_{2} \in\left(1, q_{1}\right) \text { if } n=2, \quad q_{2}^{2}=\frac{2 n}{n+2} \text { if } n \geq 3, \\
\phi=\left(\phi_{1}, \ldots, \phi_{n}\right) \in\left(L^{2}\left(\Omega_{T}\right)\right)^{n} \\
u_{0} \in L^{2}(\Omega), \quad h \in L^{2}\left(\Gamma_{2, T}\right)
\end{gathered}
$$


and the ellipticity condition

$$
\sum_{i, j=1}^{n} a_{i j} \lambda_{i} \lambda_{i} \geq \epsilon|\lambda|^{2}, \quad x \in \bar{\Omega}, \lambda \in \mathbb{R}^{n} \text { with some } \epsilon>0 \text {, }
$$

(for the sake of simplicity we introduced an assumption for the extension of $g$ onto $\Omega_{T}$ ).

The first aim is to reformulate the problem (2)-(5) in a weak form. Let us suppose that $a_{i j} \in W_{2}^{1}(\Omega),\left(\partial / \partial x_{i}\right) \phi_{i} \in$ $L^{2}\left(\Omega_{T}\right), i=1, \ldots, n$ and (2)-(5) has a classical solution $u \in$ $W_{2}^{2,1}\left(\Omega_{T}\right)$. Then, we multiply (2) with a test function $\eta$ from the space

$$
\begin{array}{r}
\mathscr{T}\left(\Omega_{T}\right)=\left\{\eta \in L^{2}\left((0, T) ; W_{2}^{1}(\Omega)\right):\right. \\
\eta_{t} \in L^{2}\left((0, T) ; L^{2}(\Omega)\right), \\
\left.\left.\eta\right|_{\Gamma_{1, T}}=0 \text { in case } \Gamma_{1} \neq \emptyset\right\}
\end{array}
$$

and integrate by parts with respect to time and space variables. We obtain the following relation:

$$
\begin{aligned}
0= & \int_{\Omega}\left[u(x, T) \eta(x, T)-u_{0}(x) \eta(x, 0)\right] d x-\iint_{\Omega_{T}} u \eta_{t} d x d t \\
& +\iint_{\Omega_{T}}\left[\sum_{i, j=1}^{n} a_{i j}\left(u_{x_{j}}-m * u_{x_{j}}\right) \eta_{x_{i}}-a(u-m * u) \eta\right] d x d t \\
& +\iint_{\Gamma_{2, T}}(\vartheta u+h) \eta d \Gamma d t-\iint_{\Omega_{T}}(f \eta-\phi \cdot \nabla \eta) d x d t .
\end{aligned}
$$

This relation makes sense also in a more general case when $a_{i j}, \phi$ satisfies only (11) and (15) and $u$ does not have regular first-order time and second-order spatial derivatives.

We call a weak solution of the problem (2)-(5) a function from the space $\mathcal{U}\left(\Omega_{T}\right)$ that satisfies the relation (19) for any $\eta \in \mathscr{T}\left(\Omega_{T}\right)$ and in case $\Gamma_{1} \neq \emptyset$ fulfills the boundary condition (4).

Lemma 1. The following assertions are valid.

(i) $\mathcal{U}\left(\Omega_{T}\right) \hookrightarrow L^{2}\left((0, T) ; L^{q_{3}}(\Omega)\right)$ where $q_{3}=\infty$ if $n=1, q_{3} \in$ $\left(q_{1} q_{2} /\left(q_{1}-q_{2}\right), \infty\right)$ if $n=2$ and $q_{3}=2 n /(n-2)$ if $n>2$, where $q_{1}$ and $q_{2}$ are given in (12) and (14), respectively;

(ii) for any $u \in L^{2}\left((0, T) ; L^{q_{3}}(\Omega)\right)$ it holds au $\in L^{2}((0, T)$; $\left.L^{q_{2}}(\Omega)\right)$ and $\|a u\|_{L^{2}\left((0, T) ; L^{q_{2}}(\Omega)\right)} \leq C\|a\|_{L^{q_{1}}(\Omega)}\|u\|_{L^{2}\left((0, T) ; L^{q_{3}}(\Omega)\right)}$, where $C$ is a constant.

Proof. Since $\mathcal{U}\left(\Omega_{T}\right) \hookrightarrow L^{2}\left((0, T) ; W_{2}^{1}(\Omega)\right)$, the assertion (i) follows from the continuous embedding of $W_{2}^{1}(\Omega)$ in $L^{q_{3}}(\Omega)$. The assertion (ii) can be proved by means of Hölder's inequality.
Theorem 2. The problem (2)-(5) has a unique weak solution. This solution satisfies the estimate

$$
\begin{aligned}
& \|u\|_{\mathcal{U}\left(\Omega_{T}\right)} \\
& \leq C_{0}\left[\left\|u_{0}\right\|_{L^{2}(\Omega)}+\|f\|_{L^{2}\left((0, T) ; L^{q^{2}}(\Omega)\right)}+\|\phi\|_{\left(L^{2}\left(\Omega_{T}\right)\right)^{n}}\right. \\
& \left.\quad+\theta_{1}\left\{\|g\|_{L^{2}\left((0, T) ; W_{2}^{1}(\Omega)\right)}+\left\|g_{t}\right\|_{L^{2}\left(\Omega_{T}\right)}\right\}+\theta_{2}\|h\|_{L^{2}\left(\Gamma_{2, T}\right)}\right],
\end{aligned}
$$

where $\theta_{1}=0$ in case $\Gamma_{1}=\emptyset, \theta_{2}=0$ in case $\Gamma_{2}=\emptyset$ and $C_{0}$ is a constant depending on $\Omega, \Gamma_{j}, a_{i j}, a, \vartheta$ and $m$.

Proof. The assertion of the theorem in case $m=0$ is well known from the theory of parabolic equations (see, e.g., [2]). Let $\mathscr{Z}$ be the operator that assigns to the data vector $d:=$ $\left(u_{0}, f, \phi, g, h\right)$ the weak solution of the problem (2)-(5) in case $m=0$. Then it holds

$$
\|\mathscr{Z}\|_{\mathscr{U}\left(\Omega_{T}\right)} \leq \mathrm{RHS},
$$

where RHS is the right-hand side of (20).

Further, let us formulate the problem for the difference $v=u-\mathscr{Z} d$. Introducing the linear operator $\mathscr{A}$ by the formula

$$
\mathscr{A} w=\mathscr{Z}\left(0,-a m * w,-\sum_{j=1}^{n} a_{i j} m * w_{x_{j}}, 0,0\right),
$$

the weak problem (2)-(5) for the solution $u \in \mathscr{U}\left(\Omega_{T}\right)$ equivalent to the following operator equation for the quantity $v$ :

$$
v=\mathscr{A} v+\mathscr{A} \mathscr{Z} d
$$

We have to estimate $\mathscr{A}$. For this purpose, we firstly prove the following auxiliary inequality:

$$
\begin{array}{r}
\|m * y\|_{L^{2}\left((0, t) ; L^{p}(\Omega)\right)} \leq \int_{0}^{t}|m(t-\tau)|\|y\|_{L^{2}\left((0, \tau) ; L^{p}(\Omega)\right)} d \tau, \\
t \in[0, T],
\end{array}
$$

for any $p \geq 1$ and $y \in L^{2}\left((0, t) ; L^{p}(\Omega)\right)$.

Denoting $\bar{y}(t)=y(\cdot, t),\|\bar{y}(t)\|=\|\bar{y}(t)\|_{L^{p}(\Omega)}$, making use of the following property of the Bochner integral: $\left\|\int_{0}^{s} w(s, \tau) d \tau\right\|_{L^{p}(\Omega)} \leq \int_{0}^{s}\|w(s, \tau)\|_{L^{p}(\Omega)} d \tau$ for functions $w \in$ $L^{1}\left((0, T) ; L^{p}(\Omega)\right)$ and the Cauchy's inequality, the relation 
(24) can be deduced by means of the following computations:

$\|m * y\|_{L^{2}\left((0, t) ; L^{p}(\Omega)\right)}$

$$
\begin{aligned}
= & {\left[\int_{0}^{t}\left\|\int_{0}^{s} m(\tau) \bar{y}(s-\tau) d \tau\right\|_{L^{p}(\Omega)}^{2} d s\right]^{1 / 2} \leq I, \text { where } } \\
I= & {\left[\int_{0}^{t}\left(\int_{0}^{s}|m(\tau)|\|\bar{y}(s-\tau)\| d \tau\right)^{2} d s\right]^{1 / 2} } \\
= & {\left[\int_{0}^{t}|m(\tau)| \int_{\tau}^{t}\|\bar{y}(s-\tau)\| \int_{0}^{s}|m(z)|\|\bar{y}(s-z)\| d z d s d \tau\right]^{1 / 2} } \\
\leq & {\left[\int_{0}^{t}|m(\tau)|\left[\int_{\tau}^{t}\|\bar{y}(s-\tau)\|^{2} d s\right]^{1 / 2}\right.} \\
\leq & \quad\left[\int_{0}^{t}|m(\tau)|\left[\int_{\tau}^{t}\left(\int_{0}^{s}|m(z)|\|\bar{y}(s-z)\| d z\right)^{2} d s\right]^{1 / 2} d \tau\right]^{1 / 2} \\
& \left.\times\left[\int_{0}^{t}\left(\int_{0}^{s}|m(z)|\|\bar{y}(s-z)\| d z\right)^{2} d s\right]^{1 / 2} d \tau\right]^{1 / 2} \\
= & {\left[\int_{0}^{t}|m(t-\tau)|\|y\|_{L^{2}\left((0, \tau) ; L^{p}(\Omega)\right)} d \tau\right]^{1 / 2} \times I^{1 / 2} }
\end{aligned}
$$

Next, let $t \in[0, T]$ and introduce the operator

$$
P_{t} w= \begin{cases}w & \text { in } \Omega_{t} \\ 0 & \text { in } \Omega_{T} \backslash \Omega_{t} .\end{cases}
$$

Due to the causality we have $\mathscr{Z}\left(0, P_{t} f, P_{t} \phi, 0,0\right)(x, t)=$ $\mathscr{Z}(0, f, \phi, 0,0)(x, t)$ for any $(x, t) \in \Omega_{t}$. Using these relations, the continuity of the linear operator $\mathscr{Z}$, the inequality (24), and the boundedness of $a_{i j}$, we compute the following:

$$
\begin{aligned}
& \|\mathscr{A} w\|_{\mathcal{U}\left(\Omega_{t}\right)} \\
& =\left\|\mathscr{Z}\left(0,-a m * w,-\sum_{j=1}^{n} a_{i j} m * w_{x_{j}}, 0,0\right)\right\|_{\mathscr{U}\left(\Omega_{t}\right)} \\
& =\left\|\mathscr{Z}\left(0,-P_{t}[a m * w],-P_{t}\left[\sum_{j=1}^{n} a_{i j} m * w_{x_{j}}\right], 0,0\right)\right\|_{\mathscr{U}\left(\Omega_{t}\right)} \\
& \leq\left\|\mathscr{Z}\left(0,-P_{t}[a m * w],-P_{t}\left[\sum_{j=1}^{n} a_{i j} m * w_{x_{j}}\right], 0,0\right)\right\|_{\mathscr{U}\left(\Omega_{T}\right)} \\
& \leq \widehat{C}\left[\left\|P_{t}[a m * w]\right\|_{L^{2}\left((0, T) ; L^{\left.q_{2}(\Omega)\right)}\right.}+\sum_{i=1}^{n}\left\|P_{t}\left[a_{i j} m * w_{x_{j}}\right]\right\|_{L^{2}\left(\Omega_{T}\right)}\right]
\end{aligned}
$$

$$
\begin{aligned}
& =\widehat{C}\left[\|a m * w\|_{L^{2}\left((0, t) ; L^{q_{2}}(\Omega)\right)}+\sum_{i=1}^{n}\left\|a_{i j} m * w_{x_{j}}\right\|_{L^{2}\left(\Omega_{t}\right)}\right] \\
& \leq \widetilde{C} \int_{0}^{t}|m(t-\tau)|\left(\|a w\|_{L^{2}\left((0, \tau) ; L^{q_{2}}(\Omega)\right)}+\|\nabla w\|_{L^{2}\left(\Omega_{\tau}\right)}\right) d \tau,
\end{aligned}
$$

with some constants $\widehat{C}$ and $\widetilde{C}$ depending on $\Omega, \Gamma_{j}, a_{i j}, a, \vartheta$. Using Lemma 1, we obtain

$$
\|a w\|_{L^{2}\left((0, \tau) ; L^{q_{2}}(\Omega)\right)} \leq C_{1}\|a\|_{L^{q_{1}(\Omega)}}\|w\|_{L^{2}\left((0, \tau) ; W_{2}^{1}(\Omega)\right)} .
$$

Using this relation in (27), we arrive at the following basic estimate for $\mathscr{A}$ :

$$
\|\mathscr{A} w\|_{\mathscr{U}\left(\Omega_{t}\right)} \leq C_{2} \int_{0}^{t}|m(t-\tau)|\|w\|_{\mathcal{U}\left(\Omega_{\tau}\right)} d \tau, \quad t \in[0, T],
$$

where $C_{2}$ is a constant depending on $\Omega, \Gamma_{j}, a_{i j}, a, \vartheta$. Let us define the weighted norms in $\mathscr{U}\left(\Omega_{T}\right):\|v\|_{\sigma}=$ $\sup _{0<t<T} e^{-\sigma t}\|v\|_{\mathcal{U}\left(\Omega_{t}\right)}$ where $\sigma \geq 0$. The estimate (29) implies the further estimate

$$
\begin{aligned}
\|\mathscr{A} w\|_{\sigma} & \leq C_{2} \sup _{0<t<T} e^{-\sigma t} \int_{0}^{t}|m(t-\tau)|\|w\|_{\mathscr{U}\left(\Omega_{\tau}\right)} d \tau \\
& =C_{2} \sup _{0<t<T} \int_{0}^{t} e^{-\sigma(t-\tau)}|m(t-\tau)| e^{-\sigma \tau}\|w\|_{\mathcal{U}\left(\Omega_{\tau}\right)} d \tau \\
& \leq C_{2} \int_{0}^{T} e^{-\sigma s}|m(s)| d s\|w\|_{\sigma} .
\end{aligned}
$$

Since $\int_{0}^{T} e^{-\sigma s}|m(s)| d s \rightarrow 0$ as $\sigma \rightarrow \infty$, there exists $\sigma_{0}$, depending on $C_{2}$ and $m$, such that $C_{2} \int_{0}^{T} e^{-\sigma_{0} s}|m(s)| d s \leq 1 / 2$. Thus, $\|\mathscr{A} w\|_{\sigma_{0}} \leq(1 / 2)\|w\|_{\sigma_{0}}$. The operator $\mathscr{A}$ is a contraction in $\mathcal{U}\left(\Omega_{T}\right)$. This implies the existence and uniqueness assertions of the theorem.

To prove the estimate (20), we firstly deduce from (23) the inequality $\|v\|_{\sigma_{0}} \leq\|\mathscr{A} v\|_{\sigma_{0}}+\|\mathscr{A} \mathscr{E} d\|_{\sigma_{0}} \leq(1 / 2)\left[\|v\|_{\sigma_{0}}+\right.$ $\|\mathscr{Z} d\|_{\sigma_{0}}$. This implies $\|v\|_{\sigma_{0}} \leq\|\mathscr{Z} d\|_{\sigma_{0}}$. Using the equivalence relations $e^{-\sigma_{0} T}\|\cdot\|_{0} \leq\|\cdot\|_{\sigma_{0}} \leq\|\cdot\|_{0}$, where $\|\cdot\|_{0}=\|\cdot\|_{\mathcal{U}\left(\Omega_{T}\right)}$ and (21), we reach (20).

We note the upper integration bound $T$ in (19) can be released to be any number $t$ from the interval $[0, T]$. Indeed, (19) is equivalent to the following problem:

$$
\begin{gathered}
0=\int_{\Omega}\left[u(x, t) \eta(x, t)-u_{0}(x) \eta(x, 0)\right] d x-\iint_{\Omega_{t}} u \eta_{t} d x d t \\
+\iint_{\Omega_{t}}\left[\sum_{i, j=1}^{n} a_{i j}\left(u_{x_{j}}-m * u_{x_{j}}\right) \eta_{x_{i}}\right. \\
-a(u-m * u) \eta] d x d t
\end{gathered}
$$




$$
\begin{aligned}
& +\iint_{\Gamma_{2, t}}(\vartheta u+h) \eta d \Gamma d t \\
& -\iint_{\Omega_{t}}(f \eta-\phi \cdot \nabla \eta) d x d t, \quad t \in[0, T],
\end{aligned}
$$

for any $\eta \in \mathscr{T}\left(\Omega_{T}\right)$. This assertion can be proved using the standard technique defining the test function as follows:

$$
\eta^{\epsilon}(x, t)= \begin{cases}\eta(x, \tau) & \text { for } \tau \in[0, t], \\ \eta(x, \tau)\left(1-\frac{\tau-t}{\epsilon}\right) & \text { for } \tau \in(t, t+\epsilon), \\ 0 & \text { for } \tau \in[t+\epsilon, T]\end{cases}
$$

and letting the parameter $\epsilon$ to approach 0 .

Next we transform the weak direct problem (31) to a form that does not contain a time derivative of the test function $\eta$. This form enables the extension of the test space. This is useful for treatment of problems for adjoint states of quasisolutions of inverse problems in next sections.

Theorem 3. The function $u \in \mathcal{U}\left(\Omega_{T}\right)$ satisfies the relation (19) for any $\eta \in \mathscr{T}\left(\Omega_{T}\right)$ if and only if it satisfies the following relation:

$$
\begin{aligned}
0= & \int_{\Omega} u * \eta d x-\int_{\Omega} \int_{0}^{t} u_{0}(x) \eta(x, \tau) d \tau d x \\
& +\int_{\Omega} 1 *\left[\sum_{i, j=1}^{n} a_{i j}\left(u_{x_{j}}-m * u_{x_{j}}\right) * \eta_{x_{i}}\right. \\
-a(u-m * u) * \eta] d x & \\
& +\int_{\Gamma_{2}} 1 *(\vartheta u+h) * \eta d \Gamma \\
& -\int_{\Omega} 1 *\left(f * \eta-\sum_{i=1}^{n} \phi_{i} * \eta_{x_{i}}\right) d x, \quad t \in[0, T],
\end{aligned}
$$

for any $\eta \in \mathcal{U}_{0}\left(\Omega_{T}\right)$.

Here, according to the definition of the time convolution in the previous section, $1 * w(t)=\int_{0}^{t} w(\tau) d \tau$.

Proof. It is sufficient to prove that $u \in \mathcal{U}\left(\Omega_{T}\right)$ satisfies (31) for any $\eta \in \mathscr{T}\left(\Omega_{T}\right)$ if and only if it satisfies (33) for any $\eta \in$ $\mathcal{U}_{0}\left(\Omega_{T}\right)$. Suppose that $u \in \mathcal{U}\left(\Omega_{T}\right)$ satisfies (31) and choose an arbitrary $\eta \in \mathscr{T}\left(\Omega_{T}\right)$. Let $t_{1}$ be an arbitrary number on the interval $[0, T]$ and choose some function $\xi^{t_{1}} \in \mathscr{T}\left(\Omega_{T}\right)$ such that the relation

$$
\xi^{t_{1}}(x, t)=\eta\left(x, t_{1}-t\right) \quad \text { for } t \in\left[0, t_{1}\right]
$$

is valid. For instance, it is possible to define $\xi^{t_{1}}$ as a periodic function with respect to $t$, that is, $\xi^{t_{1}}(x, t)=\eta\left(x, t_{1}-t\right)$ for $t \in\left[0, t_{1}\right], \xi^{t_{1}}(x, t)=\eta\left(x, t-t_{1}\right)$ for $t \in\left[t_{1}, 2 t_{1}\right], \xi^{t_{1}}(x, t)=$ $\eta\left(x, 3 t_{1}-t\right)$ for $t \in\left[2 t_{1}, 3 t_{1}\right]$ and so on. Using the relation (31) with $\eta$ replaced by $\xi^{t_{1}}$ and setting there $t=t_{1}$ we obtain the equality

$$
0=K_{1}\left(t_{1}\right)+K_{2}\left(t_{1}\right)
$$

where

$$
\begin{aligned}
K_{1}(t)= & \int_{\Omega}\left[u(x, t) \eta(x, 0)-u_{0}(x) \eta(x, t)\right] d x \\
& +\int_{\Omega} \int_{0}^{t} u(x, \tau) \eta_{t}(x, t-\tau) d \tau d x, \\
K_{2}(t)= & \int_{\Omega}\left[\sum_{i, j=1}^{n} a_{i j}\left(u_{x_{j}}-m * u_{x_{j}}\right) * \eta_{x_{i}}\right. \\
& -a(u-m * u) * \eta] d x \\
& +\int_{\Gamma_{2}}(\vartheta u+h) * \eta d \Gamma \\
& -\int_{\Omega}\left(f * \eta-\sum_{i=1}^{n} \phi_{i} * \eta_{x_{i}}\right) d x .
\end{aligned}
$$

Note that the time derivative of $\eta$ can be removed from $K_{1}$ by integration. Indeed, let $t_{2} \in[0, T]$. Then

$$
\begin{aligned}
\int_{0}^{t_{2}} K_{1}\left(t_{1}\right) d t_{1}= & \int_{0}^{t_{2}} \int_{\Omega} u\left(x, t_{1}\right) \eta(x, 0) d x d t_{1} \\
& -\int_{0}^{t_{2}} \int_{\Omega} u_{0}(x) \eta\left(x, t_{1}\right) d x d t_{1} \\
& +\int_{0}^{t_{2}} \int_{\Omega} \int_{0}^{t_{1}} u(x, \tau) \eta_{t_{1}}\left(x, t_{1}-\tau\right) d \tau d x d t_{1} .
\end{aligned}
$$

Changing the order of the integrals over $\tau$ and $t_{1}$ in the last term, we easily obtain

$$
\begin{aligned}
\int_{0}^{t_{2}} & K_{1}\left(t_{1}\right) d t_{1} \\
= & \int_{\Omega} \int_{0}^{t_{2}} u(x, \tau) \eta\left(x, t_{2}-\tau\right) d \tau d x \\
& -\int_{0}^{t_{2}} \int_{\Omega} u_{0}(x) \eta\left(x, t_{1}\right) d x d t_{1} .
\end{aligned}
$$

Integrating now the whole equality (35) over $t_{1}$ from 0 to $t_{2}$, observing (37) and (39), and finally redenoting $t_{2}$ by $t$, we reach the desired relation (33). Summing up, we have proved that (33) holds for any $\eta \in \mathscr{T}\left(\Omega_{T}\right)$. But all terms in the righthand side of (33) are well defined for $\eta \in \mathcal{U}_{0}\left(\Omega_{T}\right)$, too. Since $\mathscr{T}\left(\Omega_{T}\right)$ is densely embedded in $\mathscr{U}_{0}\left(\Omega_{T}\right)$, we conclude that (33) holds for any $\eta \in \mathcal{U}_{0}\left(\Omega_{T}\right)$.

It remains to show that (33) implies (31). Suppose that $u \in \mathcal{U}\left(\Omega_{T}\right)$ satisfies $(33)$ and choose an arbitrary $\eta \in \mathscr{T}\left(\Omega_{T}\right)$ 
and $t_{1} \in[0, T]$. Again, let $\xi^{t_{1}}$ be a function from $\mathscr{T}\left(\Omega_{T}\right)$ such (34) is valid. Inserting $\xi^{t_{1}}$ instead of $\eta$ into (33), differentiating with respect to $t$ and setting $t=t_{1}$ we come to the relation (31). Theorem is proved.

Corollary 4. A function $u \in \mathcal{U}\left(\Omega_{T}\right)$ is a weak solution of (2)(5) if and only if it satisfies the relation (33) for any $\eta \in \mathcal{U}_{0}\left(\Omega_{T}\right)$ and in case $\Gamma_{1} \neq \emptyset$ fulfills the boundary condition (4).

\section{Inverse Problems and Quasisolutions}

In the sequel, let us pose some inverse problems for the weak solution of (2)-(5). These problems are selected in order to demonstrate the wide possibilities of the method that we will introduce in Section 5. form:

Firstly, we suppose that (2)-(5) has the following specific

$$
\begin{gathered}
u_{t}=A u-m * A u+f_{0}+\nabla \cdot \phi+\sum_{j=1}^{N} \gamma_{j}(t) \omega_{j}(x) \quad \text { in } \Omega_{T}, \\
u=u_{0} \quad \text { in } \Omega \times\{0\} \\
u=g \quad \text { in } \Gamma_{1, T}, \\
-v_{A} \cdot \nabla u+m * v_{A} \cdot \nabla u=\vartheta u+h+v \cdot \phi \quad \text { in } \Gamma_{2, T}
\end{gathered}
$$

where $\omega=\left(\omega_{1}, \ldots, \omega_{N}\right)$ is unknown. The coefficients and other given functions $f_{0}, \phi, u_{0}, g, h$ are assumed to satisfy (11)-(17). Moreover, $\gamma \in\left(L^{2}(0, T)\right)^{N}$ is prescribed.

$I P 1$. Find the vector $\omega \in\left(L^{2}(\Omega)\right)^{N}$ such that the weak solution of (40) satisfies the following instant additional conditions:

$$
u\left(x, T_{i}\right)=u_{T_{i}}(x), \quad x \in \Omega, i=1,2, \ldots, N
$$

where $0<T_{1}<T_{2}<\cdots<T_{N} \leq T$ and $u_{T_{i}} \in L^{2}(\Omega)$, $i=1, \ldots, N$ are given functions (observations of $u$ ).

Since $\sum_{i=1}^{N} \gamma_{j} \omega_{j} \in L^{2}\left((0, T) ; L^{2}(\Omega)\right) \subset L^{2}\left((0, T) ; L^{q_{2}}(\Omega)\right)$ for $\omega \in\left(L^{2}(\Omega)\right)^{N}$, the weak solution $u$ of $(40)$ exists in $\mathcal{U}\left(\Omega_{T}\right)$; hence, it has traces $u\left(\cdot, T_{i}\right) \in L^{2}(\Omega), i=1, \ldots, N$. In practice, the term $\sum_{j=1}^{N} \gamma_{j} \omega_{j}$ may represent an approximation of a more general function $F(x, t) \in L^{2}\left(\Omega_{T}\right)$, where $\gamma_{j}, j=1,2, \ldots$ form a basis in $L^{2}(0, T)$.

Further, let $u_{0}$ also be unknown.

$I P 2$. Find the vector $\omega \in\left(L^{2}(\Omega)\right)^{N}$ and $u_{0} \in L^{2}(\Omega)$ such that the weak solution of (40) satisfies the following integral additional conditions:

$$
\int_{0}^{T} \kappa_{i}(x, t) u(x, t) d t=v_{i}(x), \quad x \in \Omega, i=1,2, \ldots, N+1,
$$

where $v_{i} \in L^{2}(\Omega), i=1, \ldots, N+1$ are given observation functions and $\kappa_{i}, i=1, \ldots, N$ are given weights that satisfy the following condition:

$$
\begin{array}{r}
\left|\kappa_{i}(x, t)\right| \leq \widehat{\kappa}(t) \quad \text { in } \Omega_{T}, i=1, \ldots, N+1 \\
\text { with some } \widehat{\kappa} \in L^{2}(0, T) .
\end{array}
$$

Note that the integral $\int_{0}^{T} \kappa_{i}(\cdot, t) u(\cdot, t) d t$ in (42) belongs to $L^{2}(\Omega)$ for any $\omega \in\left(L^{2}(\Omega)\right)^{N}$ and $u_{0} \in L^{2}(\Omega)$. Indeed, for such $\omega$ and $u_{0}$ it holds $u \in \mathcal{U}\left(\Omega_{T}\right) \subset L^{2}\left(\Omega_{T}\right)$, which implies

$$
\left\|\int_{0}^{T} \kappa_{i}(x, t) u(x, t) d t\right\|_{L^{2}(\Omega)} \leq\|\widehat{\kappa}\|_{L^{2}(0, T)}\|u\|_{L^{2}\left(\Omega_{T}\right)}<\infty .
$$

In practice, the weights $\kappa_{i}$ are usually concentrated in neighborhoods of some fixed values of time $t=T_{i}$.

Finally, let us pose a nonlinear inverse problem for the coefficient $a$ and the kernel $m$. Assume that $n \in\{1 ; 2 ; 3\}$. Then any coefficient $a$ that belongs to $L^{2}(\Omega)$ satisfies (12). Moreover, let us set $q_{1}=2$ if $n=2$ and $\Gamma_{2} \not \equiv \emptyset$. The other coefficients and the given functions $u_{0}, f, \phi, g, h$ are assumed to satisfy (11)-(17).

$I P 3$. Find $a \in L^{2}(\Omega)$ and $m \in L^{1}(0, T)$ such that the weak solution of (2)-(5) satisfies the following integral additional conditions:

$$
\begin{gathered}
u(x, T)=u_{T}(x), \quad x \in \Omega \\
\int_{\Gamma_{2}} \kappa(x, t) u(x, t) d \Gamma=v(t), \quad t \in(0, T),
\end{gathered}
$$

where $u_{T} \in L^{2}(\Omega), v \in L^{2}(0, T)$ are given observation functions and $\kappa$ is a given weight function such that $\kappa \in L^{\infty}((0, T)$; $\left.L^{2}\left(\Gamma_{2}\right)\right)$.

As in IP1, we can show that the trace $u(\cdot, T)$ belongs to $L^{2}(\Omega)$. Moreover, using the property $u \in \mathcal{U}\left(\Omega_{T}\right)$, the embedding of $W_{2}^{1}(\Omega)$ in $L^{2}\left(\Gamma_{2}\right)$ and Hölder's inequality, one can immediately check that the term $\int_{\Gamma_{2}} \kappa(x, \cdot) u(x, \cdot) d \Gamma$ in (45) belongs to $L^{2}(0, T)$.

Available existence, uniqueness, and stability results for IP1-IP3 require stronger smoothness of the data than imposed in the present paper. Let us cite some of these results.

In case $N=1$, the well posedness of IP1 was proved in [8]. Partial results were deduced earlier in [9]. A more general problem involving both IP1 and IP2 without the unknown $u_{0}$ in case $N=1$ was studied in [10] by means of different techniques. IP1 and IP2 in case $m=0$ and $N=1$ were treated in many papers, for example, [11-14]. The case $N>1$ is open even if $m=0$. Inverse problems to determine $m$ with given $a$ were studied in a number of papers, for example, [7, 15-23]. The problem for $a$ with given $m$ was treated in [8].

In the present paper, we will deal with quasisolutions of IP1-IP3 and related cost functionals. Denote $\mathscr{Z}_{1}=\left(L^{2}(\Omega)\right)^{N}$. 
Let $M \subseteq \mathscr{Z}_{1}$. The quasi-solution of IP1 in the set $M$ is an element $\omega^{*} \in \arg \min _{\omega \in M} J_{1}(\omega)$, where $J_{1}$ is the following cost functional

$$
J_{1}(\omega)=\sum_{i=1}^{N}\left\|u\left(x, T_{i} ; \omega\right)-u_{T_{i}}(x)\right\|_{L^{2}(\Omega)}^{2},
$$

and $u(x, t ; \omega)$ is the solution of $(40)$ that corresponds to a fixed element $\omega$.

Similarly, let $M \subseteq \mathscr{Z}_{2}:=\left(L^{2}(\Omega)\right)^{N+1}$. The quasi-solution of IP2 in the set $M$ is $z^{*} \in \arg \min _{z \in M} J_{2}(z)$, where $J_{2}$ is the cost functional

$$
J_{2}(z)=\sum_{i=1}^{N+1}\left\|\int_{0}^{T} \kappa_{i}(x, t) u(x, t ; z) d t-v_{i}(x)\right\|_{L^{2}(\Omega)}^{2},
$$

and $u(x, t ; z)$ is the weak solution of $(40)$ that corresponds to a given vector $z=\left(\omega, u_{0}\right)$.

Finally, defining $M \subseteq \mathscr{Z}_{3}:=L^{2}(\Omega) \times L^{2}(0, T)$, the quasisolution of IP3 in $M$ is an element $z^{*} \in \arg \min _{z \in M} J_{3}(z)$, where $J_{3}$ is the cost functional

$$
\begin{aligned}
J_{3}(z)= & \left\|u(x, T ; z)-u_{T}(x)\right\|_{L^{2}(\Omega)}^{2} \\
& +\left\|\int_{\Gamma_{2}} \kappa(x, t) u(x, t ; z) d \Gamma-v(t)\right\|_{L^{2}(0, T)}^{2},
\end{aligned}
$$

and $u(x, t ; z)$ is the weak solution of the direct problem (2)(5) corresponding to given $z=(a, m)$. Here, we restricted the space for the unknown $m$ to $L^{2}(0, T)$, because we will seek for the Fréchet derivative of $J_{3}$ in a Hilbert space. Moreover, the kernel of the second addend corresponding to $m$ in the representation formula of $J_{3}^{\prime}(90)$ is an element of $L^{2}(0, T)$ and in general does not belong to the adjoint space $L^{\infty}(0, T)$.

According to the above-mentioned arguments, the functionals $J_{k}, k=1,2,3$, are well-defined in $\mathscr{Z}_{1}, \mathscr{Z}_{2}$, and $\mathscr{Z}_{3}$, respectively.

\section{The Fréchet Derivatives of Cost Functionals of Inverse Problems}

5.1. General Procedure. Suppose that the solution $u$ of the direct problem depends on a vector of parameters $p$ that has to be determined in an inverse problem making use of certain measurements of $u$. Let the quasi-solution of the inverse problem be sought by a method involving the Fréchet derivative of the cost functional (i.e., some gradient-type method). Usually in practice, a solution of a proper adjoint problem is used to represent the Fréchet derivative.

We introduce a general procedure to deduce such adjoint problems. Assume that $\Delta u$ is the difference of solutions of the direct problem corresponding to a difference of the vector of the parameters $\Delta p$. More precisely, we suppose that $\Delta u$ is a solution of the following problem:

$$
\begin{gathered}
\Delta u_{t}=A \Delta u-m * A \Delta u+f^{\dagger}+\nabla \cdot \phi^{\dagger} \\
\Delta u=\Delta u_{0} \quad \text { in } \Omega \times\{0\} \\
\Delta u=0 \quad \text { in } \Gamma_{1, T} \\
-v_{A} \cdot \nabla \Delta u+m * v_{A} \cdot \nabla \Delta u=\vartheta \Delta u+h^{\dagger}+v \cdot \phi^{\dagger}
\end{gathered}
$$

with some data $f^{\dagger}, \phi^{\dagger}, \Delta u_{0}, h^{\dagger}$ depending on $\Delta p$. We restrict ourselves to the case when the Dirichlet boundary condition of the state $u$ is independent of $p$. Therefore, the condition (51) for $\Delta u$ is homogeneous.

In practice, the adjoint parabolic problems are usually formulated as backward problems. In our context, it is better to pose adjoint problems in the forward form. The involved memory term with $m$ is defined via a forward convolution and from the practical viewpoint, it is preferable to have the direct and adjoint problems in a similar form (e.g., to simplify parallelisation of computations).

More precisely, let the adjoint state $\psi$ be a solution of the following problem:

$$
\begin{gathered}
\psi_{t}=A \psi-m * A \psi+f^{\circ}+\nabla \cdot \phi^{\circ} \quad \text { in } \Omega_{T}, \\
\psi=u^{\circ} \quad \text { in } \Omega \times\{0\}, \\
\psi=0 \quad \text { in } \Gamma_{1, T}, \\
-v_{A} \cdot \nabla \psi+m * v_{A} \cdot \nabla \psi=\vartheta \psi+h^{\circ}+v \cdot \phi^{\circ} \quad \text { in } \Gamma_{2, T},
\end{gathered}
$$

where $f^{\circ}, \phi^{\circ}, u^{\circ}$, and $h^{\circ}$ are some data depending on $\Delta u$ and the cost functional under consideration.

Assume that the quadruplets $f^{\dagger}, \phi^{\dagger}, \Delta u_{0}, h^{\dagger}$, and $f^{\circ}, \phi^{\circ}$, $u^{\circ}, h^{\circ}$ satisfy the conditions (14)-(16). Then, due to Theorem 2 , the problems (49)-(52) and (53) have unique weak solutions in the space $\mathscr{U}\left(\Omega_{T}\right)$. Actually, we have $\Delta u, \psi \in \mathcal{U}_{0}\left(\Omega_{T}\right)$ because of the homogeneous boundary conditions on $\Gamma_{1, T}$.

Let us write the relation (33) for $\Delta u$ and use the test function $\eta=\psi$. Then we obtain for any $t \in[0, T]$

$$
\begin{aligned}
0= & \int_{\Omega} \Delta u * \psi d x-\int_{\Omega} \int_{0}^{t} \Delta u_{0}(x) \psi(x, \tau) d \tau d x \\
& +\int_{\Omega} 1 *\left[\sum_{i, j=1}^{n} a_{i j}\left(\Delta u_{x_{j}}-m * \Delta u_{x_{j}}\right) * \psi_{x_{i}}\right. \\
-a(\Delta u-m * \Delta u) * \psi \eta] d x & \\
& +\int_{\Gamma_{2}} 1 *\left(\vartheta \Delta u+h^{\dagger}\right) * \psi d \Gamma \\
& -\int_{\Omega} 1 *\left(f^{\dagger} * \psi-\sum_{i=1}^{n} \phi_{i}^{\dagger} * \psi_{x_{i}}\right) d x .
\end{aligned}
$$


Secondly, let us write this relation for $\psi$ and use the test function $\eta=\Delta u$. Then we have for any $t \in[0, T]$

$$
\begin{aligned}
0= & \int_{\Omega} \psi * \Delta u d x-\int_{\Omega} \int_{0}^{t} u^{\circ}(x) \Delta u(x, \tau) d \tau d x \\
& +\int_{\Omega} 1 *\left[\sum_{i, j=1}^{n} a_{i j}\left(\psi_{x_{j}}-m * \psi_{x_{j}}\right) * \Delta u_{x_{i}}\right. \\
-a(\psi-m * \psi) * \Delta u] d x & \\
& +\int_{\Gamma_{2}} 1 *\left(9 \psi+h^{\circ}\right) * \Delta u d \Gamma \\
& -\int_{\Omega} 1 *\left(f^{\circ} * \Delta u-\sum_{i=1}^{n} \phi_{i}^{\circ} * \Delta u_{x_{i}}\right) d x .
\end{aligned}
$$

Subtracting (54) from (55), using the commutativity of the convolution, the symmetricity relations $a_{i j}=a_{j i}$ and differentiating with respect to $t$, we arrive at the following basic equality that can be used in various inverse problems:

$$
\begin{aligned}
& \int_{\Omega} u^{\circ}(x) \Delta u(x, t) d x-\int_{\Gamma_{2}} h^{\circ} * \Delta u d \Gamma \\
& \quad+\int_{\Omega}\left(f^{\circ} * \Delta u-\sum_{i=1}^{n} \phi_{i}^{\circ} * \Delta u_{x_{i}}\right) d x \\
& =\int_{\Omega} \Delta u_{0}(x) \psi(x, t) d x-\int_{\Gamma_{2}} h^{\dagger} * \psi d \Gamma \\
& \quad+\int_{\Omega}\left(f^{\dagger} * \psi-\sum_{i=1}^{n} \phi_{i}^{\dagger} * \psi_{x_{i}}\right) d x, \quad t \in[0, T] .
\end{aligned}
$$

\subsection{Derivative of $J_{1}$}

Theorem 5. The functional $J_{1}$ is the Fréchet differentiable in $\left(L^{2}(\Omega)\right)^{N}$ and

$$
\begin{aligned}
& J_{1}^{\prime}(\omega) \Delta \omega \\
& \quad=\sum_{j=1}^{N} \int_{\Omega} \sum_{i=1}^{N} \int_{0}^{T_{i}} \gamma_{j}(t) \psi_{i}\left(x, T_{i}-t ; \omega\right) d t \Delta \omega_{j}(x) d x,
\end{aligned}
$$

where $\psi_{i} \in \mathcal{U}\left(\Omega_{T_{i}}\right), i=1, \ldots, N$, are the unique $\omega$-dependent weak solutions of the following problems:

$$
\begin{gathered}
\psi_{i, t}=A \psi_{i}-m * A \psi_{i} \quad \text { in } \Omega_{T_{i}}, \\
\psi_{i}=2\left[u\left(x, T_{i} ; \omega\right)-u_{T_{i}}(x)\right] \quad \text { in } \Omega \times\{0\} \\
\psi_{i}=0 \quad \text { in } \Gamma_{1, T_{i}}, \\
-v_{A} \cdot \nabla \psi_{i}+m * v_{A} \cdot \nabla \psi_{i}=\vartheta \psi_{i} \quad \text { in } \Gamma_{2, T_{i}},
\end{gathered}
$$

$i=1, \ldots, N$.
Proof. Let us fix some $\omega, \Delta \omega \in\left(L^{2}(\Omega)\right)^{N}$. One can immediately check that it holds

$$
\begin{aligned}
J_{1}(\omega & +\Delta \omega)-J_{1}(\omega) \\
= & 2 \sum_{i=1}^{N} \int_{\Omega}\left[u\left(x, T_{i} ; \omega\right)-u_{T_{i}}(x)\right] \Delta u\left(x, T_{i} ; \omega\right) d x \\
& +\sum_{i=1}^{N} \int_{\Omega} \Delta u\left(x, T_{i} ; \omega\right)^{2} d x,
\end{aligned}
$$

where $\Delta u(x, t ; \omega)=u(x, t ; \omega+\Delta \omega)-u(x, t ; \omega) \in \mathcal{U}_{0}\left(\Omega_{T}\right)$ is the weak solution of the following problem:

$$
\begin{gathered}
\Delta u_{t}=A \Delta u-m * A \Delta u+\sum_{j=1}^{N} \gamma_{j} \Delta \omega_{j} \quad \text { in } \Omega_{T}, \\
\Delta u=0 \quad \text { in } \Omega \times\{0\}, \\
\Delta u=0 \quad \text { in } \Gamma_{1, T}, \\
-v_{A} \cdot \nabla \Delta u+m * v_{A} \cdot \nabla \Delta u=\vartheta \Delta u \quad \text { in } \Gamma_{2, T} .
\end{gathered}
$$

Applying the estimate (20) to the solution of this problem we deduce the following estimate for the second term in the right-hand side of (59):

$$
\begin{aligned}
& \sum_{i=1}^{N} \int_{\Omega} \Delta u\left(x, T_{i} ; \omega\right)^{2} d x \leq n\|\Delta u\|_{u\left(\Omega_{T}\right)}^{2} \\
& \leq C_{0}^{2} n\left\|\sum_{j=1}^{N} \gamma_{j} \Delta \omega_{j}\right\|_{L^{2}\left((0, T) ; L^{q_{2}}(\Omega)\right)}^{2} \leq C_{4}\|\omega\|_{\left(L^{2}(\Omega)\right)^{N}}^{2}
\end{aligned}
$$

with some constant $C_{4}$. This implies that $J_{1}$ is the Fréchet differentiable and the first term in the right-hand side of (59) represents the Fréchet derivative, that is,

$$
J_{1}^{\prime}(\omega) \Delta \omega=\sum_{i=1}^{N} \sigma_{i}
$$

$$
\text { with } \sigma_{i}=2 \int_{\Omega}\left[u\left(x, T_{i} ; \omega\right)-u_{T_{i}}(x)\right] \Delta u\left(x, T_{i} ; \omega\right) d x \text {. }
$$

Further, let us use the method presented in Section 5.1 to deduce the proper adjoint problems. Comparing (60) with (49)-(52) we see that $f^{\dagger}=\sum_{j=1}^{N} \gamma_{j} \Delta \omega_{j}, \phi^{\dagger}=\Delta u_{0}=h^{\dagger}=0$. Therefore, the relation (56) has the form

$$
\begin{aligned}
\int_{\Omega} u^{\circ}(x) \Delta u(x, t) d x-\int_{\Gamma_{2}} h^{\circ} * \Delta u d \Gamma \\
\quad+\int_{\Omega}\left(f^{\circ} * \Delta u-\sum_{i=1}^{n} \phi_{i}^{\circ} * \Delta u_{x_{i}}\right) d x \\
=\sum_{j=1}^{N} \int_{\Omega} \gamma_{j} \Delta \omega_{j} * \psi d x, \quad t \in[0, T] .
\end{aligned}
$$


In order to deduce a formula for the component $\sigma_{i}$ in the quantity $J_{1}^{\prime}(\omega) \Delta \omega$, we set $u^{\circ}=u_{i}^{\circ}=2\left[u\left(x, T_{i} ; \omega\right)-u_{T_{i}}(x)\right]$, $h^{\circ}=f^{\circ}=\phi^{\circ}=0$ and $t=T_{i}$ in (63). Then we immediately have

$$
\sigma_{i}=\left.\sum_{j=1}^{N} \int_{\Omega} \gamma_{j} \Delta \omega_{j} * \psi_{i} d x\right|_{t=T_{i}},
$$

where according to (53) and the definition of $u_{i}^{\circ}, h^{\circ}, \phi^{\circ}$, the function $\psi_{i}$ is the weak solution of the problem (58) in the domain $\Omega_{T}$ instead of $\Omega_{T_{i}}$. Due to Theorem 2, this problem has a unique solution. From (62) and (64) we obtain (57). The latter formula contains the values of $\psi_{i}$ in $\Omega_{T_{i}}$. Therefore, we can restrict the problem (58) from $\Omega_{T}$ to $\Omega_{T_{i}}$.

To use the formula (57) one has to solve $N$ weak problems for the functions $\psi_{i}$ in domains $\Omega_{T_{i}}$. In the following theorem, we will show that computational work related to the evaluation of the Fréchet derivative can be considerably reduced. Actually, it is sufficient to solve $N$ weak problems in the smaller domains $\Omega_{T_{i}-T_{i-1}}, i=1, \ldots, N$. Here, $T_{0}=0$.

Theorem 6. The Fréchet derivative of the functional $J_{1}$ can also be written in the form

$$
\begin{aligned}
& J_{1}^{\prime}(\omega) \Delta \omega \\
& =\sum_{j=1}^{N} \int_{\Omega} \sum_{l=1}^{N} \int_{T_{l-1}}^{T_{l}} \gamma_{j}(t) \beta_{l}\left(x, T_{l}-t, \omega\right) d t \Delta \omega_{j}(x) d x,
\end{aligned}
$$

where $\beta_{l} \in \mathcal{U}_{0}\left(\Omega_{T_{l}-T_{l-1}}\right)$ are the unique $\omega$-dependent weak solutions of the following sequence of recursive problems in the domains $\Omega_{T_{l}-T_{l-1}}$ :

$$
\begin{gathered}
\beta_{l, t}=A \beta_{l}-m * A \beta_{l}-a f^{l}-\nabla \cdot \Phi^{l} \text { in } \Omega_{T_{l}-T_{l-1}}, \\
\beta_{l}=u_{0}^{l} \quad \text { in } \Omega \times\{0\}, \\
\beta_{l}=0 \quad \text { in } \Gamma_{1, T_{l}-T_{l-1}},
\end{gathered}
$$

$$
-v_{A} \cdot \nabla \beta_{l}+m * v_{A} \cdot \nabla \beta_{l}=\vartheta \beta_{l}-v \cdot \Phi^{l} \text { in } \Gamma_{2, T_{l}-T_{l-1}},
$$

where $l=N, N-1, \ldots, 2,1$. Here,

$$
u_{0}^{l}(x)=2\left[u\left(x, T_{l} ; \omega\right)-u_{T_{l}}(x)\right]+\Theta_{l} \beta_{l+1}\left(x, T_{l+1}-T_{l}\right)
$$

and the function $f^{l}$ and the vector $\Phi^{l}$ are defined via $\beta_{N}$, $\beta_{N-1}, \ldots, \beta_{l+1}$ as follows:

$$
\begin{aligned}
& f^{l}=\Theta_{l} \sum_{k=l}^{N-1} \int_{0}^{T_{k+1}-T_{k}} m\left(T_{k}-T_{l}+t+\tau\right) \beta_{k+1} \\
& \times\left(x, T_{k+1}-T_{k}-\tau\right) d \tau, \\
& \Phi^{l}=\left(\Phi_{1}^{l}, \ldots, \Phi_{n}^{l}\right), \Phi_{i}^{l}=\sum_{j=1}^{n} a_{i j}\left(\partial / \partial x_{j}\right) f^{l} \text { and } \Theta_{N}=0, \Theta_{l}= \\
& 1 \text { for } l<N .
\end{aligned}
$$

Proof. Firstly, let us check that (66) indeed have unique weak solutions $\beta_{l}$ in $\mathscr{U}_{0}\left(\Omega_{T_{l}-T_{l-1}}\right)$. To this end we can use Theorem 2. For the problem $\beta_{N}$ this is immediate, because the initial condition of the problem for $\beta_{N}$ belongs to $L^{2}(\Omega)$ and other equations in this problem are homogeneous. Further, we use the induction. Choose some $l$ in the range $N>l \geq 1$ and suppose that $\beta_{k+1} \in \mathscr{U}_{0}\left(\Omega_{T_{k+1}-T_{k}}\right)$ for all $k$ such that $N-1 \geq k \geq l$. The aim is to us to show that then the problem for $\beta_{l}$ has a unique weak solution in $\mathcal{U}_{0}\left(\Omega_{T_{l}-T_{l-1}}\right)$. Let us represent the $k$ th addend in (68) in the form

$$
\begin{aligned}
I_{k} & =\int_{0}^{T_{k+1}-T_{k}} m\left(T_{k}-T_{l}+t+\tau\right) \beta_{k+1}\left(x, T_{k+1}-T_{k}-\tau\right) d \tau \\
& =\int_{t}^{T_{k+1}-T_{k}+t} m\left(T_{k}-T_{l}+\tau\right) \beta_{k+1}\left(x, T_{k+1}-T_{k}+t-\tau\right) d \tau .
\end{aligned}
$$

For any $k$ in the range $N-1 \geq k \geq l$ we have

$$
\begin{gathered}
\left\|I_{k}\right\|_{L^{2}\left(\left(0, T_{l}-T_{l-1}\right) ; W_{2}^{1}(\Omega)\right)}^{2}\left[\sum _ { | \alpha | \leq 1 } \int _ { 0 } ^ { T _ { l } - T _ { l - 1 } } \left[\int_{k+1}^{T_{k}-T_{k}+t}\left|m\left(T_{k}-T_{l}+\tau\right)\right|\right.\right. \\
\left.\cdot\left\|D_{x}^{\alpha} \beta_{k+1}\left(\cdot, T_{k+1}-T_{k}+t-\tau\right)\right\|_{L^{2}(\Omega)} d \tau\right]^{2} d t \\
=\sum_{|\alpha| \leq 1} \int_{0}^{T_{l}-T_{l-1}}\left[\int_{t}^{T_{k+1}-T_{k}+t} \int_{0}^{T_{k+1}-T_{k}+t}\left|m\left(T_{k}-T_{l}+\tau\right)\right| z_{k, \alpha}\right. \\
=\sum_{|\alpha| \leq 1} \int_{T_{k+1}-T_{k}}^{T_{l}-T_{l-1}+T_{k+1}-T_{k}}\left[\int_{k+1}^{t} m_{k}(\tau) z_{k, \alpha}(t-\tau) d \tau\right]^{2} d t
\end{gathered}
$$

where $z_{k, \alpha}(t)=\left\|D_{x}^{\alpha} \beta_{k+1}(\cdot, t)\right\|_{L^{2}(\Omega)}$ for $t \in\left[0, T_{k+1}-T_{k}\right]$, $z_{k, \alpha}(t)=0$ for $t \notin\left[0, T_{k+1}-T_{k}\right]$ and $m_{k}(t)=\left|m\left(T_{k}-T_{l}+t\right)\right|$. Since $m \in L^{1}(0, T)$ and $\beta_{k+1} \in L^{2}\left(\left(0, T_{k+1}-T_{k}\right) ; W_{2}^{1}(\Omega)\right)$, we have $m_{k} \in L^{1}\left(0, T_{l}-T_{l-1}+T_{k+1}-T_{k}\right)$ and $z_{k, \alpha} \in L^{2}\left(0, T_{l}-\right.$ $\left.T_{l-1}+T_{k+1}-T_{k}\right)$. Due to the Young's theorem for convolutions, we get $m_{k} * z_{k, \alpha} \in L^{2}\left(0, T_{l}-T_{l-1}+T_{k+1}-T_{k}\right)$. Therefore, $\left\|I_{k}\right\|_{L^{2}\left(\left(0, T_{l}-T_{l-1}\right) ; W_{2}^{1}(\Omega)\right)}^{2}<\infty$. This implies that $f_{l}$ belongs to $L^{2}\left(\left(0, T_{l}-T_{l-1}\right) ; W_{2}^{1}(\Omega)\right)$. From the latter relation and $a_{i j} \in$ $L^{\infty}(\Omega)$ we immediately have $\Phi^{l} \in\left(L^{2}\left(\Omega_{T_{l}-T_{l-1}}\right)\right)^{n}$. Using the embedding theorem and Lemma 1 we see that $a f^{l}$ satisfies the property (14). Finally, the initial condition $u_{0}^{l}$ belongs to $L^{2}(\Omega)$, because $u-u_{T_{l}}, \beta_{l+1} \in C\left(\left[0, T_{l+1}-T_{l}\right], L^{2}(\Omega)\right)$. All assumptions of Theorem 2 are satisfied for the problem for $\beta_{l}$. Consequently, it possesses a unique weak solution in $\mathcal{U}_{0}\left(\Omega_{T_{l}-T_{l-1}}\right)$. 
Secondly, let us define the functions

$$
\beta_{l}^{*}(x, t)=\sum_{i=l}^{N} \psi_{i}\left(x, T_{i}-T_{l}+t\right) \quad \text { for }(x, t) \in \Omega_{T_{l}-T_{l-1}},
$$

where $l=1 \ldots, N$ and $\psi_{i}$ are the solutions of (58). We are going to show that $\beta_{l}=\beta_{l}^{*}, l=1, \ldots, N$. From the definition of $\beta_{l}^{*}$ using the value of $\psi_{l}(x, 0)$ and simple computations, we immediately get

$$
\begin{array}{r}
\beta_{l}^{*}(x, 0)=2\left[u\left(x, T_{l} ; \omega\right)-u_{T_{l}}(x)\right]+\Theta_{l} \beta_{l+1}^{*}\left(x, T_{l+1}-T_{l}\right), \\
l=1, \ldots, N .
\end{array}
$$

Let us fix $l=1, \ldots, N$ and choose some $\eta \in \mathscr{T}\left(\Omega_{T_{l}-T_{l-1}}\right)$. We continue $\eta$ by the formulae $\eta(x, t)=\eta\left(x, T_{l}-T_{l-1}\right)$ for $t>T_{l}-T_{l-1}$ and $\eta(x, t)=\eta(x, 0)$ for $t<0$. Further, let us define $\eta_{i}(x, t)=\eta\left(x, T_{l}-T_{i}+t\right)$ where $i=l, \ldots, N$. By the definition, it holds $\eta_{i} \in \mathscr{T}\left(\Omega_{T_{i}}\right)$.

Let us write down the weak form (31) for the problem for $\psi_{i}(58)$ with the test function $\eta_{i}$. We fix some $t \in\left[0, T_{l}-\right.$ $\left.T_{l-1}\right]$ and compute the difference of this weak problem with $t$ replaced by $T_{i}-T_{l}+t$ and $t$ replaced by $T_{i}-T_{l}$ and take the sum over $i=l, \ldots, N$. This results in the following expression:

$$
0=Z_{1}+Z_{2}+Z_{3}+Z_{4}
$$

where

$$
\begin{gathered}
Z_{1}=\sum_{i=l}^{N} \int_{\Omega}\left[\psi_{i}\left(x, T_{i}-T_{l}+t\right) \eta_{i}\left(x, T_{i}-T_{l}+t\right)\right. \\
\left.-\psi_{i}\left(x, T_{i}-T_{l}\right) \eta_{i}\left(x, T_{i}-T_{l}\right)\right] d x, \\
Z_{2}=\sum_{i=l}^{N} \iint_{\Omega_{T_{i}-T_{l}+t} \mid \Omega_{T_{i}-T_{l}}}\left[-\psi_{i} \eta_{i, t}\right. \\
\left.+\sum_{s, j=1}^{n} a_{s j} \psi_{i, x_{j}} \eta_{i, x_{s}}-a \psi_{i} \eta_{i}\right] d x d t \\
+\sum_{i=l}^{N} \iint_{\Gamma_{2, T_{i}-T_{l}+t} \backslash \Gamma_{2, T_{i}-T_{l}}} 9 \psi_{i} \eta_{i} d \Gamma d t, \\
Z_{3}=\sum_{i=l}^{N} \iint_{\Omega_{T_{i}-T_{l}+t} \mid \Omega_{T_{i}-T_{l}}} a m * \psi_{i} \eta_{i} d x d t, \\
Z_{4}=-\sum_{i=l}^{N} \iint_{\Omega_{T_{i}-T_{l}+t} \mid \Omega_{T_{i}-T_{l}}} \sum_{s, j=1}^{n} a_{s j} m * \psi_{i, x_{j}} \eta_{i, x_{s}} d x d t .
\end{gathered}
$$

Using the definitions of $\eta$ and $\beta_{l}^{*}$ and the formula (72), we have

$$
\begin{aligned}
& Z_{1}=\int_{\Omega}[\left.\beta_{l}^{*}(x, t) \eta(x, t)-\beta_{l}^{*}(x, 0) \eta(x, 0)\right] d x \\
&=\int_{\Omega}\left[\beta_{l}^{*}(x, t) \eta(x, t)\right. \\
&- \\
& \quad\left\{2\left[u\left(x, T_{l} ; \omega\right)-u_{T_{l}}(x)\right]+\Theta_{l} \beta_{l+1}^{*}\left(x, T_{l+1}-T_{l}\right)\right\} \\
&\quad \times \eta(x, 0)] d x .
\end{aligned}
$$

Similarly, using the definitions of $\eta$ and $\beta_{l}^{*}$ and changing the variable of integration in $Z_{2}$, we deduce

$$
\begin{aligned}
Z_{2}= & \iint_{\Omega_{t}}\left[-\beta_{l}^{*} \eta_{t}+\sum_{s, j=1}^{n} a_{s j} \beta_{l}^{*} \eta_{x_{s}}-a \beta_{l}^{*} \eta\right] d x d t \\
& +\iint_{\Gamma_{2, t}} \vartheta \beta_{l}^{*} \eta d \Gamma d t .
\end{aligned}
$$

By the change of variable, the quantity $Z_{3}$ is transformed to

$$
Z_{3}=\iint_{\Omega_{t}} a(x) \sum_{i=l}^{N}\left(m * \psi_{i}\right)\left(x, T_{i}-T_{l}+t\right) \eta(x, t) d x d t .
$$

Let us consider the term $\sum_{i=l}^{N}\left(m * \psi_{i}\right)\left(x, T_{i}-T_{l}+t\right)$ in the latter formula. We compute

$$
\begin{aligned}
& \sum_{i=l}^{N}\left(m * \psi_{i}\right)\left(x, T_{i}-T_{l}+t\right) \\
& =\sum_{i=l}^{N} \int_{0}^{T_{i}-T_{l}+t} m(\tau) \psi_{i}\left(x, T_{i}-T_{l}+t-\tau\right) d \tau \\
& =\int_{0}^{t} m(\tau) \sum_{i=l}^{N} \psi_{i}\left(x, T_{i}-T_{l}+t-\tau\right) d \tau \\
& \quad+\sum_{i=l}^{N} \int_{t}^{T_{i}-T_{l}+t} m(\tau) \psi_{i}\left(x, T_{i}-T_{l}+t-\tau\right) d \tau \\
& =\int_{0}^{t} m(\tau) \beta_{l}^{*}(x, t-\tau) d \tau \\
& \quad+\sum_{i=l}^{N} \sum_{k=l}^{i-1} \int_{0}^{T_{k+1}-T_{k}} m\left(T_{k}-T_{l}+t+\tau\right) \psi_{i}\left(x, T_{i}-T_{k}-\tau\right) d \tau \\
& =\left(m * \beta_{l}^{*}\right)(x, t) \\
& \quad+\sum_{k=l}^{N-1} \int_{0}^{T_{k+1}-T_{k}} m\left(T_{k}-T_{l}+t+\tau\right) \\
& \quad \times \sum_{i=k+1}^{N} \psi_{i}\left(x, T_{i}-T_{k}-\tau\right) d \tau
\end{aligned}
$$




$$
\begin{aligned}
& =\left(m * \beta_{l}^{*}\right)(x, t) \\
& \quad+\sum_{k=l}^{N-1} \int_{0}^{T_{k+1}-T_{k}} m\left(T_{k}-T_{l}+t+\tau\right) \\
& \quad \times \beta_{k+1}^{*}\left(x, T_{k+1}-T_{k}-\tau\right) d \tau .
\end{aligned}
$$

Thus, (77) reads

$$
\begin{aligned}
& Z_{3} \\
& =\iint_{\Omega_{t}} a m * \beta_{l}^{*} \eta d x d t \\
& \quad+\iint_{\Omega_{t}} a\left[\sum_{k=l}^{N-1} \int_{0}^{T_{k+1}-T_{k}} m\left(T_{k}-T_{l}+t+\tau\right)\right. \\
& \left.\quad \times \beta_{k+1}^{*}\left(x, T_{k+1}-T_{k}-\tau\right) d \tau\right] \eta d x d t .
\end{aligned}
$$

Using similar computations, we obtain

$$
\begin{array}{r}
Z_{4}=-\iint_{\Omega_{t}} \sum_{s, j=1}^{n} a_{s j} m * \beta_{l, x_{j}}^{*} \eta_{x_{s}} d x d t-\iint_{\Omega_{t}} \sum_{s, j=1}^{n} a_{s j} \\
\times\left[\sum_{k=l}^{N-1} \int_{0}^{T_{k+1}-T_{k}} m\left(T_{k}-T_{l}+t+\tau\right)\right. \\
\left.\quad \times \beta_{k+1, x_{j}}^{*}\left(x, T_{k+1}-T_{k}-\tau\right) d \tau\right] \eta_{x_{s}} d x d t .
\end{array}
$$

Plugging (75), (76), (79), and (80) into (73), we arrive at a certain weak problem for $\beta_{l}^{*}$ that coincides with the weak problem for $\beta_{l}$. Moreover, since $\psi_{i} \in \mathcal{U}_{0}\left(\Omega_{T_{i}}\right)$, from (71) we see that $\beta_{l}^{*} \in \mathscr{U}_{0}\left(\Omega_{T_{l}-T_{l-1}}\right)$. But we have shown the uniqueness of the weak solutions of the problems for $\beta_{l}$ in $\mathcal{U}_{0}\left(\Omega_{T_{l}-T_{l-1}}\right)$. This implies $\beta_{l}^{*}=\beta_{l}$.

Finally, from (57), we have

$$
\begin{aligned}
J_{1}^{\prime}(\omega) \Delta \omega & =\sum_{j=1}^{N} \int_{\Omega} \sum_{i=1}^{N} \int_{0}^{T_{i}} \gamma_{j}(t) \psi_{i}\left(x, T_{i}-t\right) d t \Delta \omega_{j}(x) d x \\
& =\sum_{j=1}^{N} \int_{\Omega} \sum_{i=1}^{N} \sum_{l=1}^{i} \int_{T_{l-1}}^{T_{l}} \gamma_{j}(t) \psi_{i}\left(x, T_{i}-t\right) d t \Delta \omega_{j}(x) d x .
\end{aligned}
$$

Changing here the order of sums over $i$ and $l$ and observing (71) with $\beta_{l}^{*}$ replaced by $\beta_{l}$, we obtain (65). The proof is complete.

\subsection{Derivative of $J_{2}$}

Theorem 7. The functional $J_{2}$ is the Fréchet differentiable in $\left(L^{2}(\Omega)\right)^{N+1}$ and

$$
\begin{aligned}
J_{2}^{\prime}(z) \Delta z= & \sum_{j=1}^{N} \int_{\Omega} \int_{0}^{T} \gamma_{j}(t) \psi(x, T-t ; z) d t \Delta \omega_{j}(x) d x \\
& +\int_{\Omega} \psi(x, T ; z) \Delta u_{0}(x) d x
\end{aligned}
$$

where $\psi \in \mathcal{U}\left(\Omega_{T}\right)$ is the unique $z$-dependent weak solution of the following problem:

$$
\begin{aligned}
& \psi_{t}=A \psi-m * A \psi \\
& +2 \sum_{i=1}^{N+1} \kappa_{i}(x, T-t) \\
& \times\left[\int_{0}^{T} \kappa_{i}(x, \tau) u(x, \tau ; z) d \tau-v_{i}(x)\right] \quad \text { in } \Omega_{T} \\
& \psi=0 \quad \text { in } \Omega \times\{0\} \\
& \psi=0 \quad \text { in } \Gamma_{1, T}, \\
& -v_{A} \cdot \nabla \psi+m * v_{A} \cdot \nabla \psi=\vartheta \psi \quad \text { in } \Gamma_{2, T}
\end{aligned}
$$

Proof. Let us fix some $\Delta z=\left(\Delta \omega, \Delta u_{0}\right) \in\left(L^{2}(\Omega)\right)^{N+1}$. It holds

$$
\begin{aligned}
J_{2}(z+\Delta z)-J_{2}(z) \\
=2 \sum_{i=1}^{N+1} \int_{\Omega} \int_{0}^{T} \kappa_{i}(x, t) \\
\quad \times\left[\int_{0}^{T} \kappa_{i}(x, \tau) u(x, \tau ; z) d \tau-v_{i}(x)\right] \\
\quad \times \Delta u(x, t ; z) d t d x \\
\quad+\sum_{i=1}^{N+1} \int_{\Omega}\left[\int_{0}^{T} \kappa_{i}(x, t) \Delta u(x, t ; z) d t\right]^{2} d x,
\end{aligned}
$$

where $\Delta u(x, t ; z)=u(x, t ; z+\Delta z)-u(x, t ; z) \in \mathcal{U}_{0}\left(\Omega_{T}\right)$ is the weak solution of the following problem:

$$
\begin{gathered}
\Delta u_{t}=A \Delta u-m * A \Delta u+\sum_{j=1}^{N} \gamma_{j} \Delta \omega_{j} \quad \text { in } \Omega_{T}, \\
\Delta u=\Delta u_{0} \quad \text { in } \Omega \times\{0\}, \\
\Delta u=0 \quad \text { in } \Gamma_{1, T}, \\
-v_{A} \cdot \nabla \Delta u+m * v_{A} \cdot \nabla \Delta u=\vartheta \Delta u \quad \text { in } \Gamma_{2, T} .
\end{gathered}
$$


Using (43), the Cauchy inequality and estimate (20) from Theorem 2 for the problem of $\Delta u(x, t ; z)$, we come to the estimate

$$
\begin{aligned}
& \left|\sum_{i=1}^{N+1} \int_{\Omega}\left[\int_{0}^{T} \kappa_{i}(x, t) \Delta u(x, t ; z) d t\right]^{2} d x\right| \\
& \quad \leq(N+1)\|\widehat{\kappa}\|_{L^{2}(0, T)}^{2}\|\Delta u\|_{L^{2}\left(\Omega_{T}\right)}^{2} \\
& \quad \leq C_{5}(N+1)\|\widehat{\kappa}\|_{L^{2}(0, T)}^{2}\|\Delta u\|_{\mathcal{U}\left(\Omega_{T}\right)}^{2} \leq C_{6}\|\Delta z\|_{\left(L^{2}(\Omega)\right)^{N+1}}^{2},
\end{aligned}
$$

with some constants $C_{5}$ and $C_{6}$. Therefore, $J_{2}$ is the Fréchet differentiable and the first term in the right-hand side of (84) represents the Fréchet derivative, that is,

$$
\begin{aligned}
J_{2}^{\prime}(z) \Delta z= & 2 \sum_{i=1}^{N+1} \int_{\Omega} \int_{0}^{T} \kappa_{i}(x, t) \\
& \times\left[\int_{0}^{T} \kappa_{i}(x, \tau) u(x, \tau ; z) d \tau-v_{i}(x)\right] \\
& \times \Delta u(x, t ; z) d t d x
\end{aligned}
$$

Comparing (85) with (49)-(52), we see that $f^{\dagger}=\sum_{j=1}^{N} \gamma_{j} \Delta \omega_{j}$, $\phi^{\dagger}=h^{\dagger}=0$. Consequently, the relation (56) has the form

$$
\begin{aligned}
& \int_{\Omega} u^{\circ}(x) \Delta u(x, t) d x-\int_{\Gamma_{2}} h^{\circ} * \Delta u d \Gamma \\
& \quad+\int_{\Omega}\left(f^{\circ} * \Delta u-\sum_{i=1}^{n} \phi_{i}^{\circ} * \Delta u_{x_{i}}\right) d x \\
& =\int_{\Omega} \Delta u_{0}(x) \psi(x, t) d x \\
& \quad+\sum_{j=1}^{N} \int_{\Omega} \gamma_{j} \Delta \omega_{j} * \psi d x, \quad t \in[0, T] .
\end{aligned}
$$

To deduce a formula for $J_{2}^{\prime}(z) \Delta z$, we define

$$
f^{\circ}=2 \sum_{i=1}^{N+1} \kappa_{i}(x, T-t)\left[\int_{0}^{T} \kappa_{i}(x, \tau) u(x, \tau ; z) d \tau-v_{i}(x)\right]
$$

$u^{\circ}=h^{\circ}=\phi^{\circ}=0$ and $t=T$ in (88). Then from (87) and (88), we obtain (82), where due to (53), $\psi_{i}$ is the weak solution of the problem (83). In view of Theorem 2 , this problem has a unique solution in $\mathscr{U}\left(\Omega_{T}\right)$.

\subsection{Derivative of $\mathrm{J}_{3}$}

Theorem 8. The functional $J_{3}$ is the Fréchet differentiable in $L^{2}(\Omega) \times L^{2}(0, T)$ and

$$
\begin{aligned}
J_{3}^{\prime}(z) \Delta z= & \int_{\Omega}[(u-m * u) * \psi](x, T ; z) \Delta a(x) d x \\
& +\int_{0}^{T} \int_{\Omega}\left[\sum_{i, j=1}^{n} a_{i j} u_{x_{j}} * \psi_{x_{i}}-a u * \psi\right] \\
& \times(x, T-t ; z) d x \Delta m(t) d t,
\end{aligned}
$$

where $\psi \in \mathscr{U}_{0}\left(\Omega_{T}\right)$ is the unique $z$-dependent weak solution of the problem

$$
\begin{gathered}
\psi_{t}=A \psi-m * A \psi \quad \text { in } \Omega_{T}, \\
\psi=2\left[u(x, T ; z)-u_{T}(x)\right] \quad \text { in } \Omega \times\{0\} \\
\psi=0 \quad \text { in } \Gamma_{1, T}, \\
-v_{A} \cdot \nabla \psi+m * v_{A} \cdot \nabla \psi=\vartheta \psi \\
-2 \kappa(x, T-t) \\
\times\left[\int_{\Gamma_{2}} \kappa(y, T-t) u(y, T-t ; z) d \Gamma-v(T-t)\right] \quad \text { in } \Gamma_{2, T} .
\end{gathered}
$$

Proof. Due to $u(x, t ; z) \in \mathcal{U}\left(\Omega_{T}\right), \kappa \in L^{\infty}\left((0, T) ; L^{2}\left(\Gamma_{2}\right)\right)$, $v \in L^{2}(0, T)$, and $u_{T} \in L^{2}(\Omega)$, the problem (91) satisfies the assumptions of Theorem 2 . Therefore, it has a unique weak solution in $\mathscr{U}_{0}\left(\Omega_{T}\right)$.

Let $\Delta z=(\Delta a, \Delta m) \in L^{2}(\Omega) \times L^{2}(0, T)$ and define $\widetilde{\Delta} u=$ $u(x, t ; z+\Delta z)-u(x, t ; z)$. We split $\widetilde{\Delta} u$ as follows: $\widetilde{\Delta} u=\Delta u+\widehat{\Delta} u$, where $\Delta u$ is the weak solution of the following problem:

$$
\begin{aligned}
& \Delta u_{t}=A \Delta u-m * A \Delta u+\Delta a[u-m * u]-\Delta m * a u \\
& -\nabla \cdot\left[\Delta m * \sum_{j=1}^{n} a_{i j} u_{x_{j}}\right] \quad \text { in } \Omega_{T} \text {, } \\
& \Delta u=0 \quad \text { in } \Omega \times\{0\}, \\
& \Delta u=0 \quad \text { in } \Gamma_{1, T} \text {, } \\
& -v_{A} \cdot \nabla \Delta u+m * v_{A} \cdot \nabla \Delta u \\
& =\vartheta \Delta u-v \cdot\left[\Delta m * \sum_{j=1}^{n} a_{i j} u_{x_{j}}\right] \quad \text { in } \Gamma_{2, T} .
\end{aligned}
$$

In view of Lemma $1(\mathrm{i}), u \in \mathcal{U}\left(\Omega_{T}\right), m \in L^{1}(0, T)$, and the Young's theorem, it holds $u-m * u \in L^{2}\left((0, T) ; L^{q_{3}}(\Omega)\right)$. 
Therefore, Lemma 1(ii) implies

$$
\begin{aligned}
\|\Delta a[u-m * u]\|_{L^{2}\left((0, T) ; L^{q_{2}}(\Omega)\right)} & \leq C_{8}(u, m)\|\Delta a\|_{L^{q_{1}}(\Omega)} \\
& \leq C_{9}(u, m)\|\Delta a\|_{L^{2}(\Omega)},
\end{aligned}
$$

where $C_{8}$ and $C_{9}$ are some constants depending on $u, m$. Moreover, since $u \in L^{2}\left((0, T) ; W_{2}^{1}(\Omega)\right)$, by Young's inequality we have also

$$
\begin{aligned}
\left\|\Delta m * \sum_{j=1}^{n} a_{i j} u_{x_{j}}\right\|_{\left(L^{2}\left(\Omega_{T}\right)\right)^{n}} & \leq C_{10}(u)\|\Delta m\|_{L^{1}(0, T)} \\
& \leq C_{11}(u)\|\Delta m\|_{L^{2}(0, T)},
\end{aligned}
$$

with some constants $C_{10}$ and $C_{11}$ depending on $u$. The obtained estimates show that assumptions of Theorem 2 are satisfied for the problem (92) and it indeed has a unique weak solution $\Delta u \in \mathscr{U}\left(\Omega_{T}\right)$. Moreover, applying the relation (20) from Theorem 2, we get

$$
\begin{aligned}
\|\Delta u\|_{\mathcal{U}\left(\Omega_{T}\right)} & \leq C_{12}(m, u)\left[\|\Delta a\|_{L^{2}(\Omega)}+\|\Delta m\|_{L^{2}(0, T)}\right] \\
& =C_{12}(m, u)\|\Delta z\|
\end{aligned}
$$

where $C_{12}(m, u)$ is a constant depending on $m, u$.

Further, writing the problem for $\widetilde{\Delta} u$ and subtracting the problem for $\Delta u$, we obtain the following problem for $\widehat{\Delta} u$ :

$$
\begin{gathered}
\widehat{\Delta} u_{t}=A \widehat{\Delta} u-m * A \widehat{\Delta} u+f+\widehat{f}+\nabla \cdot \phi+\nabla \cdot \widehat{\phi} \quad \text { in } \Omega_{T}, \\
\widehat{\Delta} u=0 \quad \text { in } \Omega \times\{0\}, \\
\widehat{\Delta} u=0 \quad \text { in } \Gamma_{1, T}, \\
-v_{A} \cdot \nabla \widehat{\Delta} u+m * v_{A} \cdot \nabla \widehat{\Delta} u=\vartheta \widehat{\Delta} u+v \cdot \phi+v \cdot \widehat{\phi} \quad \text { in } \Gamma_{2, T},
\end{gathered}
$$

where

$$
\begin{aligned}
& f=\Delta a \Delta u-(m+\Delta m) * \Delta a \Delta u-\Delta m * \Delta a u-\Delta m * a \Delta u, \\
& \widehat{f}=\Delta a \widehat{\Delta} u-(m+\Delta m) * \Delta a \widehat{\Delta} u-\Delta m * a \widehat{\Delta} u, \\
& \phi=-\Delta m * \sum_{j=1}^{n} a_{i j} \Delta u_{x_{j}}, \quad \widehat{\phi}=-\Delta m * \sum_{j=1}^{n} a_{i j} \widehat{\Delta} u_{x_{j}} .
\end{aligned}
$$

Using again Lemma 1 and the Young's inequality, we deduce the estimates

$$
\begin{aligned}
& \|f\|_{L^{2}\left((0, T) ; L^{\left.q_{2}(\Omega)\right)}\right.} \\
& \leq C_{13}\left\{\left[\|\Delta a\|_{L^{2}(\Omega)}+\left\{\|m\|_{L^{2}(0, T)}+\|\Delta m\|_{L^{2}(0, T)}\right\}\|\Delta a\|_{L^{2}(\Omega)}\right.\right. \\
& \left.+\|\Delta m\|_{L^{2}(0, T)}\|a\|_{L^{2}(\Omega)}\right]\|\Delta u\|_{\mathcal{U}\left(\Omega_{T}\right)} \\
& \left.+\|u\|_{\mathcal{U}\left(\Omega_{T}\right)}\|\Delta a\|_{L^{2}(\Omega)}\|\Delta m\|_{L^{2}(0, T)}\right\} \\
& \leq C_{14}(z, u)\left\{\left[\|\Delta z\|+\|\Delta z\|^{2}\right]\|\Delta u\|_{\mathscr{U}\left(\Omega_{T}\right)}+\|\Delta z\|^{2}\right\} \\
& \|\hat{f}\|_{L^{2}\left((0, T) ; L^{\left.q_{2}(\Omega)\right)}\right.} \leq C_{15}(z)\left[\|\Delta z\|+\|\Delta z\|^{2}\right]\|\widehat{\Delta} u\|_{\mathscr{U}\left(\Omega_{T}\right)^{\prime}} \\
& \|\phi\|_{\left(L^{2}\left(\Omega_{T}\right)\right)^{n}} \leq C_{16}\|\Delta m\|_{L^{2}(0, T)}\|\Delta u\|_{\mathcal{U}\left(\Omega_{T}\right)} \\
& \leq C_{16}\|\Delta z\|\|\Delta u\|_{\mathcal{U}\left(\Omega_{T}\right)}, \\
& \|\widehat{\phi}\|_{\left(L^{2}\left(\Omega_{T}\right)\right)^{n}} \leq C_{17}\|\Delta z\|\|\widehat{\Delta} u\|_{\mathcal{U}\left(\Omega_{T}\right)}
\end{aligned}
$$

with some constants $C_{13} \cdots C_{17}$. Therefore, applying the relation (20) to the solution of the problem (96) we obtain

$$
\begin{aligned}
& \|\widehat{\Delta} u\|_{\mathscr{U}\left(\Omega_{T}\right)} \\
& \leq C_{18}(z, u) \\
& \quad \times\left\{\left[\|\Delta z\|+\|\Delta z\|^{2}\right]\left\{\|\Delta u\|_{\mathcal{U}\left(\Omega_{T}\right)}+\|\widehat{\Delta} u\|_{\mathcal{U}\left(\Omega_{T}\right)}\right\}+\|\Delta z\|^{2}\right\},
\end{aligned}
$$

with some constant $C_{18}$. In case $\|\Delta z\|$ is small enough, that is,

$$
\|\Delta z\|+\|\Delta z\|^{2} \leq \frac{1}{2 C_{18}(z, u)}
$$

we have

$$
\begin{aligned}
\|\widehat{\Delta} u\|_{\mathcal{U}\left(\Omega_{T}\right)} \leq & 2 C_{18}(z, u) \\
& \times\left\{\left[\|\Delta z\|+\|\Delta z\|^{2}\right]\|\Delta u\|_{\mathcal{U}\left(\Omega_{T}\right)}+\|\Delta z\|^{2}\right\} .
\end{aligned}
$$

In view of (95), this implies

$$
\|\widehat{\Delta} u\|_{\mathcal{U}_{\left(\Omega_{T}\right)}} \leq C_{19}(z, u)\left[\|\Delta z\|^{2}+\|\Delta z\|^{3}\right]
$$

with a constant $C_{19}$.

Similarly, for the solution of the problem (92), we deduce the estimate

$$
\|\Delta u\|_{\mathcal{u}\left(\Omega_{T}\right)} \leq C_{20}(z, u)\|\Delta z\|
$$

with a constant $C_{20}$. 
Now, we write the difference of $J_{3}$ in the following form:

$$
\begin{aligned}
& J_{3}(z+\Delta z)-J_{3}(z) \\
& =2 \int_{\Omega}\left[u(x, T ; z)-u_{T}(x)\right] \Delta u(x, T ; z) d x \\
& \quad+2 \int_{0}^{T} \int_{\Gamma_{2}} \kappa(x, t)\left[\int_{\Gamma_{2}} \kappa(y, t) u(y, t ; z) d \Gamma-v(t)\right] \\
& \quad \times \Delta u(x, t ; z) d \Gamma d t+\Theta,
\end{aligned}
$$

where

$$
\begin{aligned}
\Theta= & 2 \int_{\Omega}\left[u(x, T ; z)-u_{T}(x)\right] \widehat{\Delta} u(x, T ; z) d x \\
& +2 \int_{0}^{T} \int_{\Gamma_{2}} \kappa(x, t)\left[\int_{\Gamma_{2}} \kappa(y, t) u(y, t ; z) d \Gamma-v(t)\right] \\
& \times \widehat{\Delta} u(x, t ; z) d \Gamma d t \\
& +\int_{\Omega}\{(\Delta u+\widehat{\Delta} u)(x, T ; z)\}^{2} d x \\
& +\int_{0}^{T}\left\{\int_{\Gamma_{2}} \kappa(x, t)(\Delta u+\widehat{\Delta} u)(x, t ; z) d \Gamma\right\}^{2} d t .
\end{aligned}
$$

Using (102), (103), and the property $\kappa \in L^{\infty}\left((0, T) ; L^{2}\left(\Gamma_{2}\right)\right)$, we obtain the estimate $|\Theta| \leq C_{21}(z, u) \sum_{j=2}^{6}\|\Delta z\|^{j}$ in case $(100)$. This shows that $J_{3}$ is the Fréchet differentiable and

$$
\begin{aligned}
J_{3}^{\prime}(z) \Delta z= & 2 \int_{\Omega}\left[u(x, T ; z)-u_{T}(x)\right] \Delta u(x, T ; z) d x \\
& +2 \int_{0}^{T} \int_{\Gamma_{2}} \kappa(x, t) \\
& \times\left[\int_{\Gamma_{2}} \kappa(y, t) u(y, t ; z) d \Gamma-v(t)\right] \\
& \times \Delta u(x, t ; z) d \Gamma d t .
\end{aligned}
$$

Finally, let us prove (90) and (91). Comparing (92) with (49)-(52), we see that $f^{\dagger}=\Delta a[u-m * u]-\Delta m * a u, \phi_{i}^{\dagger}=$ $-\Delta m * \sum_{j=1}^{n} a_{i j} u_{x_{j}}$ and $\Delta u_{0}=h^{\dagger}=0$. Thus, (56) reads

$$
\begin{gathered}
\int_{\Omega} u^{\circ}(x) \Delta u(x, t) d x-\int_{\Gamma_{2}} h^{\circ} * \Delta u d \Gamma \\
\quad+\int_{\Omega}\left(f^{\circ} * \Delta u-\sum_{i=1}^{n} \phi_{i}^{\circ} * \Delta u_{x_{i}}\right) d x \\
=\int_{\Omega}\left(\begin{array}{c}
\{\Delta a[u-m * u]-\Delta m * a u\} * \psi \\
+\Delta m * \sum_{i, j=1}^{n} a_{i j} u_{x_{j}} * \psi_{x_{i}}
\end{array}\right) d x, \quad t \in[0, T] .
\end{gathered}
$$

In order to obtain a formula for the right-hand side in (106), we set $u^{\circ}=2\left[u(x, T ; z)-u_{T}(x)\right]$,

$$
\begin{aligned}
h^{\circ}(x, t)= & -2 \kappa(x, T-t) \\
\times & {\left[\int_{\Gamma_{2}} \kappa(y, T-t) u(y, T-t ; z) d \Gamma-v(T-t)\right], }
\end{aligned}
$$

$f^{\circ}=\phi^{\circ}=0$ and $t=T$. Then, we obtain (90), where in view of (53) the function $\psi$ is the weak solution of (91).

\section{Further Aspects of Minimisation}

6.1. Existence of Quasisolutions. For the convenience, we will use also the symbol $z$ to denote the argument $\omega$ of $J_{1}$.

Theorem 9. (i) Let $k \in\{1 ; 2\}$ and $M \subset \mathscr{Z}_{k}$ be bounded, closed, and convex. Then, IPk has a quasi-solution in $M$. The set of quasisolutions is closed and convex.

(ii) Let $k \in\{1 ; 2 ; 3\}$ and $M \subset \mathscr{Z}_{3}$ be compact. Then IPk has a quasi-solution in $M$.

Proof. Let us prove (i). The existence assertion follows from Weierstrass existence theorem (see [24, Section 2.5, Theorem $2 \mathrm{D}]$ ) once we have proved that $J_{k}$ is weakly sequentially lower semicontinuous in $\mathscr{F}$, that is,

$$
J_{k}(z) \leq \liminf _{n \rightarrow \infty} J_{k}\left(z_{n}\right) \quad \text { as } z_{n} \rightarrow z \text { in } \mathscr{Z}_{k}
$$

But (109) follows from the continuity and convexity of $J_{k}$ [24]. The convexity of $J_{k}$ can be immediately deduced making use of the linearity of the ingredient $u(x, t ; z)$ with respect to $z$ inside the quadratic functional $J_{k}$ (for similar computations see [25, Theorem 2]). The closedness of the set of quasisolutions is again a direct consequence of the continuity of $J_{k}$. The convexity of the set of solutions follows from the convexity of $J_{k}$.

Next, we prove (ii). Let $m=\inf _{z \in M} J_{k}(z)$ and $z_{l} \in M$ be the minimising sequence, that is, $\lim J_{k}\left(z_{l}\right)=m$. By the compactness, there exists a subsequence $z_{l_{j}} \in M$ such that $\lim z_{l_{j}}=z^{*} \in M$. Due to the continuity of $J_{k}$ we have $\lim J_{k}\left(z_{l_{j}}\right)=J_{k}\left(z^{*}\right)$. Thus, $J_{k}\left(z^{*}\right)=m$. This proves (ii).

In practice, the compact set $M$ may be a bounded and closed finite-dimensional subset of $\mathscr{Z}_{k}$. The proof of weak lower semicontinuity of $J_{3}$ may be harder because this functional is not convex.

6.2. Discretisation and Minimisation. Let us consider the penalised discrete problems

$$
z^{\dagger} \in \arg \min _{z \in \mathscr{F}_{k, L}} \Phi_{k, L}(z), \quad \Phi_{k, L}=\Pi_{L}(z)+J_{k}(z),
$$

where $k \in\{1 ; 2 ; 3\}, \mathscr{Z}_{k, L}$ is an $L$-dimensional subspace of $Z_{k}(L \in\{1,2, \ldots\})$ and $\Pi_{L}$ is a penalty function related to 
the set $M_{L}=P_{L} M$ with $P_{L}$ being the orthogonal projection onto $\mathscr{Z}_{k, L}$. The general assumptions for $\Pi_{L}$ are

$\Pi_{L}$-accretive, convex, Fréchet differentiable,

$$
\Pi_{L}^{\prime} \text {-uniformly Lipschitz continuous in } \mathscr{Z}_{k, L} \text {. }
$$

Theorem 10. The problem (110) has a solution.

Proof. The proof repeats the proof of the statement (ii) of Theorem 9, because in view of the accretivity of $\Phi_{k, L}$, a minimizing sequence is bounded and in a finite-dimensional space any bounded sequence is compact.

The Fréchet derivative of $\Phi_{k, L}$, that is, $\Phi_{k, L}^{\prime}(z)=\Pi_{L}^{\prime}(z)+$ $J_{k}^{\prime}(z) \in \mathscr{Z}_{k, L}^{*}=\mathscr{Z}_{k, L}$ can be identified by a certain element in $\mathscr{Z}_{k, L}$, that is,

$$
\Phi_{k, L}^{\prime}(z) \Delta z=\left\langle\Phi_{k, L}^{\prime}(z), \Delta z\right\rangle_{\mathscr{I}_{k}} \quad \forall \Delta z \in \mathscr{Z}_{k, L},
$$

where $\langle\cdot, \cdot\rangle_{\mathscr{Z}_{k}}$ is the inner product of $\mathscr{Z}_{k}$. In particular, the addend $J_{k}^{\prime}(z)$ is identical to the element $P_{L} w_{k}(z)$ where $w_{k}(z)$ is the kernel of the functional $J_{k}^{\prime}(z)$. Thus, by virtue of (57), (65), (82), and (90), it holds

$$
\begin{aligned}
w_{1}(z)= & \left(\left.\sum_{i=1}^{N} \int_{0}^{T_{i}} \gamma_{j}(t) \psi_{i}\left(\cdot, T_{i}-t ; z\right) d t\right|_{j=1, \ldots, N}\right) \\
& =\left(\left.\sum_{l=1}^{N} \int_{T_{l-1}}^{T_{l}} \gamma_{j}(t) \beta_{l}\left(\cdot, T_{l}-t ; z\right) d t\right|_{j=1, \ldots, N}\right), \\
w_{2}(z)= & \left(\left.\int_{0}^{T} \gamma_{j}(t) \psi(\cdot, T-t ; z) d t\right|_{j=1, \ldots, N}, \psi(\cdot, T ; z)\right), \\
w_{3}(z)= & \left(\int_{\Omega}\left[\sum_{i, j=1}^{n} a_{i j} u_{x_{j}} * \psi_{x_{i}}-a u * \psi\right](x, T-\cdot) d x\right) .
\end{aligned}
$$

In $w_{1}$, the functions $\psi_{i}$ and $\beta_{l}$ are the $z$ - (or, equivalently, $\omega-$ ) dependent weak solutions of the problems (58) and (66), respectively. In $w_{2}$ the function $\psi$ is the weak solution of (83) and in $w_{3}$ the functions $u$ and $\psi$ are the $z$-dependent weak solutions of (2)-(5) and (91), respectively.

Example 11. Consider the case $k=1$. Let $M=\left\{z \in \mathscr{Z}_{1}\right.$ : $\|z\| \leq \rho\}$, where $\rho>0$. Further, let $\xi_{j}, j=1,2, \ldots$, be an orthonormal basis in $L^{2}(\Omega)$ and $\mathscr{Z}_{1, L}=\left(\operatorname{span}\left(\xi_{1}, \ldots, \xi_{L}\right)\right)^{N}$. Then $J_{1}^{\prime}(z)$ is in $\mathscr{Z}_{1, L}$ identical to the element

$$
\begin{aligned}
P_{L} w_{1}(z)=\left(\sum_{i=1}^{L} \int_{\Omega} \sum_{l=1}^{N} \int_{T_{l-1}}^{T_{l}} \gamma_{j}(t) \beta_{l}\left(x, T_{l}-t ; z\right) d t\right. \\
\left.\times\left.\xi_{i}(x) d x \xi_{i}\right|_{j=1, \ldots, N}\right) .
\end{aligned}
$$

Moreover, it holds $M_{L}=\left\{z \in \mathscr{Z}_{1, L}:\|z\| \leq \rho\right\}$. Define a convex penalty function $\Pi_{L} \in C^{\infty}[0, \infty)$ such that $\Pi_{L}(z)=0$ for $\|z\| \leq \rho$ and $\Pi_{L}(z)=d\left(\|z\|^{2}-\rho^{2}\right)$ for $\|z\| \geq \rho+\varepsilon$ with some $d, \varepsilon>0$. Then $\Pi_{L}$ satisfies (111).

Let $k \in\{1 ; 2 ; 3\}$. Choose some initial guess $z_{0} \in \mathscr{Z}_{k, L}$. Compute the approximate solutions by the gradient method

$$
z_{s+1}=z_{s}-c_{s} \Phi_{k, L}^{\prime}\left(z_{s}\right),
$$

where $s=0,1,2, \ldots$ and $c_{s}>0$.

Theorem 12. Let $k \in\{1 ; 2\}$ and $c_{s}$ be chosen by the rule

$$
\begin{aligned}
\inf _{c>0} \Phi_{k, L}\left(z_{s}-c \Phi_{k, L}^{\prime}\left(z_{s}\right)\right) & \leq \Phi_{k, L}\left(z_{s}-c_{s} \Phi_{k, L}^{\prime}\left(z_{s}\right)\right) \\
& \leq \inf _{c>0} \Phi_{k, L}\left(z_{s}-c \Phi_{k, L}^{\prime}\left(z_{s}\right)\right)+\delta_{s}
\end{aligned}
$$

where $\delta_{s} \geq 0, \sum_{s=0}^{\infty} \delta_{s}=: \delta<\infty$. Then it holds $\operatorname{dist}\left(z_{s}, S\right) \rightarrow 0$ as $s \rightarrow \infty$, where $S$ is the set of solutions of (110).

Proof. The assertion follows from Theorem 5.1.2 of [26] once we have proved that $\Phi_{k, L}^{\prime}$ is uniformly Lipschitz continuous, $\Phi_{k, L}$ is convex, and the set $M\left(z_{0}\right)=\left\{z \in \mathscr{Z}_{k, L}: \Phi_{k, L}(z) \leq\right.$ $\left.\Phi_{k, L}\left(z_{0}\right)+\delta\right\}$ is bounded. The convexity of $\Phi_{k, L}$ follows from the convexity of its addends $\Pi_{L}$ and $J_{k}$. The boundedness of $M\left(z_{0}\right)$ is a direct consequence of the accretivity of $\Phi_{k, L}$ following from the accretivity of the addend $\Pi_{L}$.

It remains to show the uniform Lipschitz continuity of $J_{k}^{\prime}$ in $\mathscr{Z}_{k, L}$ (such a property for $\Pi_{L}^{\prime}$ is assumed in (111)). Let $k=1$. Then by (113) and $J_{k}^{\prime}(z)=P_{L} w_{1}(z)$ for any $z, \tilde{z} \in \mathscr{Z}_{k, L}$, we have

$$
\begin{aligned}
\left\|J_{1}^{\prime}(\widetilde{z})-J_{1}^{\prime}(z)\right\| & \leq\left\|P_{L}\right\|\left\|w_{1}(\widetilde{z})-w_{1}(z)\right\| \\
& \leq C_{22} \sum_{i=1}^{N}\left\|\psi_{i}(\cdot, \cdot ; \widetilde{z})-\psi_{i}(\cdot, \cdot ; z)\right\|_{\mathcal{U}\left(\Omega_{T}\right)},
\end{aligned}
$$

where $C_{22}$ is a constant independent of $z$ and $\widetilde{z}$. Further, observing (58) and (40), the estimate (20) of Theorem 2 and $z=\omega$, we deduce

$$
\begin{aligned}
\left\|J_{1}^{\prime}(\widetilde{z})-J_{1}^{\prime}(z)\right\| & \leq 2 C_{22} C_{0} \sum_{i=1}^{N}\left\|u\left(\cdot, T_{i} ; \widetilde{z}\right)-u\left(\cdot, T_{i} ; z\right)\right\|_{L^{2}(\Omega)} \\
& \leq C_{23}\|u(\cdot, \cdot ; \widetilde{z})-u(\cdot, \cdot ; z)\|_{\mathcal{U}\left(\Omega_{T}\right)} \\
& \leq C_{24}\|\widetilde{z}-z\|,
\end{aligned}
$$

where $C_{23}, C_{24}$ are independent of $z$ and $\widetilde{z}$. This proves the uniform Lipschitz continuity of $J_{1}^{\prime}$. Such a property of $J_{2}^{\prime}$ can be proved in a similar manner.

The convergence of $z_{s}$ in case $k=3$ is an open issue. This case is more complex because IP3 is nonlinear and the Fréchet derivative of $J_{3}$ is not uniformly Lipschitz continuous.

The quasisolutions of IP1-IP3 are not expected to be stable with respect to the noise of the data, that is, the problems 
under consideration may be ill posed. Nevertheless, from the intuitive viewpoint, a discretisation should regularise an illposed problem. Such a property of the discretisation has been proved in many cases $[27,28]$. Alternatively, the index $s$ of the gradient method could be used as a regularization parameter (see $[29,30])$. Moreover, the addend $\Pi_{L}$ can be defined to be the stabilizing term of the Tikhonov's method instead of the penalty function, that is, $\Pi_{L}=\alpha\|z\|^{2}$, where $\alpha>0$ is the regularisation parameter. Such a $\Pi_{L}$ satisfies (111).

\section{Acknowledgments}

The paper was supported by the Estonian Science Foundation (Grant 7728), Estonian Ministry of Education and Science target financed theme SF0140011s09, and the Estonian state programme Smart Composites-Design and Manufacturing.

\section{References}

[1] H. W. Engl, M. Hanke, and A. Neubauer, Regularization of Inverse Problems, Kluwer Academic Publisher, Dodrecht, The Netherlands, 2000.

[2] O. A. Ladyženskaja, V. A. Solonnikov, and N. N. Uraltseva, Linear and Quasilinear Equations of Parabolic Type, American Mathematical Society, Providence, RI, USA, 1968.

[3] L. C. Evans, Partial Differential Equations, vol. 19 of Graduate Studies in Mathematics, American Mathematical Society, Providence, RI, USA, 2nd edition, 2010.

[4] G. Amendola, M. Fabrizio, and J. M. Golden, Thermodynamics of Materials with Memory. Theory and application, Springer, New York, NY, USA, 2012.

[5] S. A. Messaoudi, "Blow-up of solutions of a semilinear heat equation with a memory term," Abstract and Applied Analysis, no. 2, pp. 87-94, 2005.

[6] J. Prüss, Evolutionary Integral Equations and Applications, vol. 87 of Monographs in Mathematics, Birkhäuser, Boston, Mass, USA, 1993.

[7] J. Janno and L. V. Wolfersdorf, "Identification of memory kernels in general linear heat flow," Journal of Inverse and IllPosed Problems, vol. 6, no. 2, pp. 141-164, 1998.

[8] J. Janno and K. Kasemets, "A positivity principle for parabolic integro-differential equations and inverse problems with final overdetermination," Inverse Problems and Imaging, vol. 3, no. 1, pp. 17-41, 2009.

[9] A. Lorenzi and A. Prilepko, "Fredholm-type results for integrodifferential identification parabolic problems," Differential and Integral Equations, vol. 6, no. 3, pp. 535-552, 1993.

[10] A. Lorenzi and G. Mola, "Identification of unknown terms in convolution integro-differential equations in a Banach space," Journal of Inverse and Ill-Posed Problems, vol. 18, no. 3, pp. 321$355,2010$.

[11] E. Beretta and C. Cavaterra, "Identifying a space dependent coefficient in a reaction-diffusion equation," Inverse Problems and Imaging, vol. 5, no. 2, pp. 285-296, 2011.

[12] A. Hasanov, "Simultaneous determination of source terms in a linear parabolic problem from the final overdetermination: weak solution approach," Journal of Mathematical Analysis and Applications, vol. 330, no. 2, pp. 766-779, 2007.

[13] V. Isakov, "Inverse parabolic problems with the final overdetermination," Communications on Pure and Applied Mathematics, vol. 44, no. 2, pp. 185-209, 1991.
[14] A. Lorenzi and I. I. Vrabie, "An identification problem for a linear evolution equation in a Banach space and applications," Discrete and Continuous Dynamical Systems. Series S, vol. 4, no. 3, pp. 671-691, 2011.

[15] F. Colombo and A. Lorenzi, "Identification of time and space dependent relaxation kernels for materials with memory related to cylindrical domains. I, II," Journal of Mathematical Analysis and Applications, vol. 213, no. 1, pp. 32-62, 1997.

[16] M. Grasselli, "An identification problem for a linear integrodifferential equation occurring in heat flow," Mathematical Methods in the Applied Sciences, vol. 15, no. 3, pp. 167-186, 1992.

[17] J. Janno, "Determination of a time- and space-dependent heat flux relaxation function by means of a restricted Dirichletto-Neumann operator," Mathematical Methods in the Applied Sciences, vol. 27, no. 11, pp. 1241-1260, 2004.

[18] J. Janno and A. Lorenzi, "A parabolic integro-differential identification problem in a barrelled smooth domain," Zeitschrift für Analysis und ihre Anwendungen, vol. 25, no. 1, pp. 103-130, 2006.

[19] J. Janno and A. Lorenzi, "Recovering memory kernels in parabolic transmission problems in infinite time intervals: the non-accessible case," Journal of Inverse and Ill-Posed Problems, vol. 18, no. 4, pp. 433-465, 2010.

[20] J. Janno and L. v. Wolfersdorf, "Identification of weakly singular memory kernels in heat conduction," Zeitschrift für Angewandte Mathematik und Mechanik, vol. 77, no. 4, pp. 243-257, 1997.

[21] J. Janno and L. von Wolfersdorf, "An inverse problem for identification of a time- and space-dependent memory kernel of a special kind in heat conduction," Inverse Problems, vol. 15, no. 6, pp. 1455-1467, 1999.

[22] J. Janno and L. v. Wolfersdorf, "Inverse problems for memory kernels by Laplace transform methods," Zeitschrift für Analysis und ihre Anwendungen, vol. 19, no. 2, pp. 489-510, 2000.

[23] A. Lorenzi and F. Messina, "An identification problem with evolution on the boundary of parabolic type," Advances in Differential Equations, vol. 13, no. 11-12, pp. 1075-1108, 2008.

[24] E. Zeidler, Applied Functional Analysis. Main principles and their application, vol. 109 of Applied Mathematical Sciences, Springer, New York, NY, USA, 1995.

[25] K. Kasemets and J. Janno, "Reconstruction of a source term in a parabolic integro-differential equation from final data," Mathematical Modelling and Analysis, vol. 16, no. 2, pp. 199-219, 2011.

[26] F. P. Vasil'ev, Methods of Solving Extremum Problems, Nauka, Moscow, Russia, 2nd edition, 1988.

[27] B. Kaltenbacher, "Regularization by projection with a posteriori discretization level choice for linear and nonlinear ill-posed problems," Inverse Problems, vol. 16, no. 5, pp. 1523-1539, 2000.

[28] R. Plato and G. Vainikko, "On the regularization of projection methods for solving ill-posed problems," Numerische Mathematik, vol. 57, no. 1, pp. 63-79, 1990.

[29] U. Hämarik and R. Palm, "On rules for stopping the conjugate gradient type methods in ill-posed problems," Mathematical Modelling and Analysis, vol. 12, no. 1, pp. 61-70, 2007.

[30] O. Scherzer, "A convergence analysis of a method of steepest descent and a two-step algorithm for nonlinear ill-posed problems," Numerical Functional Analysis and Optimization, vol. 17, no. 1-2, pp. 197-214, 1996. 


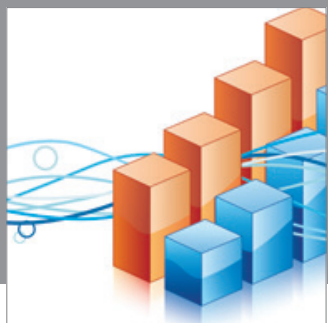

Advances in

Operations Research

mansans

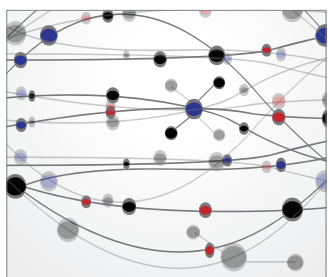

The Scientific World Journal
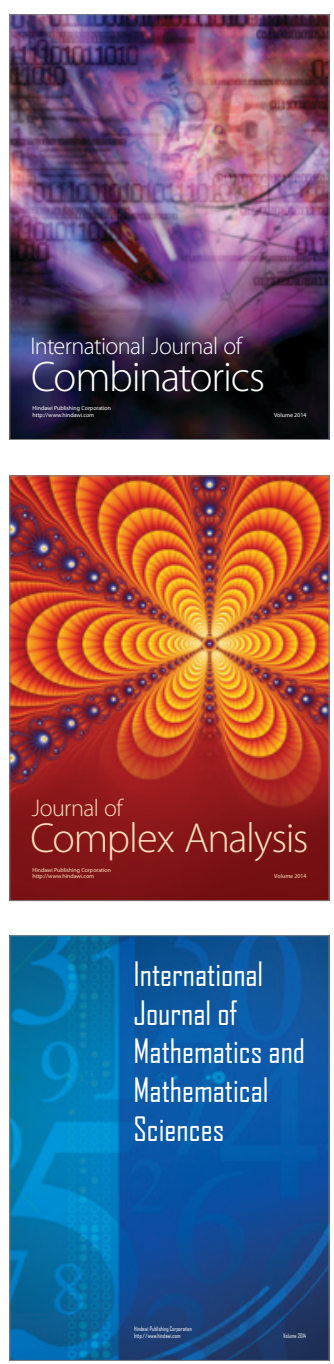
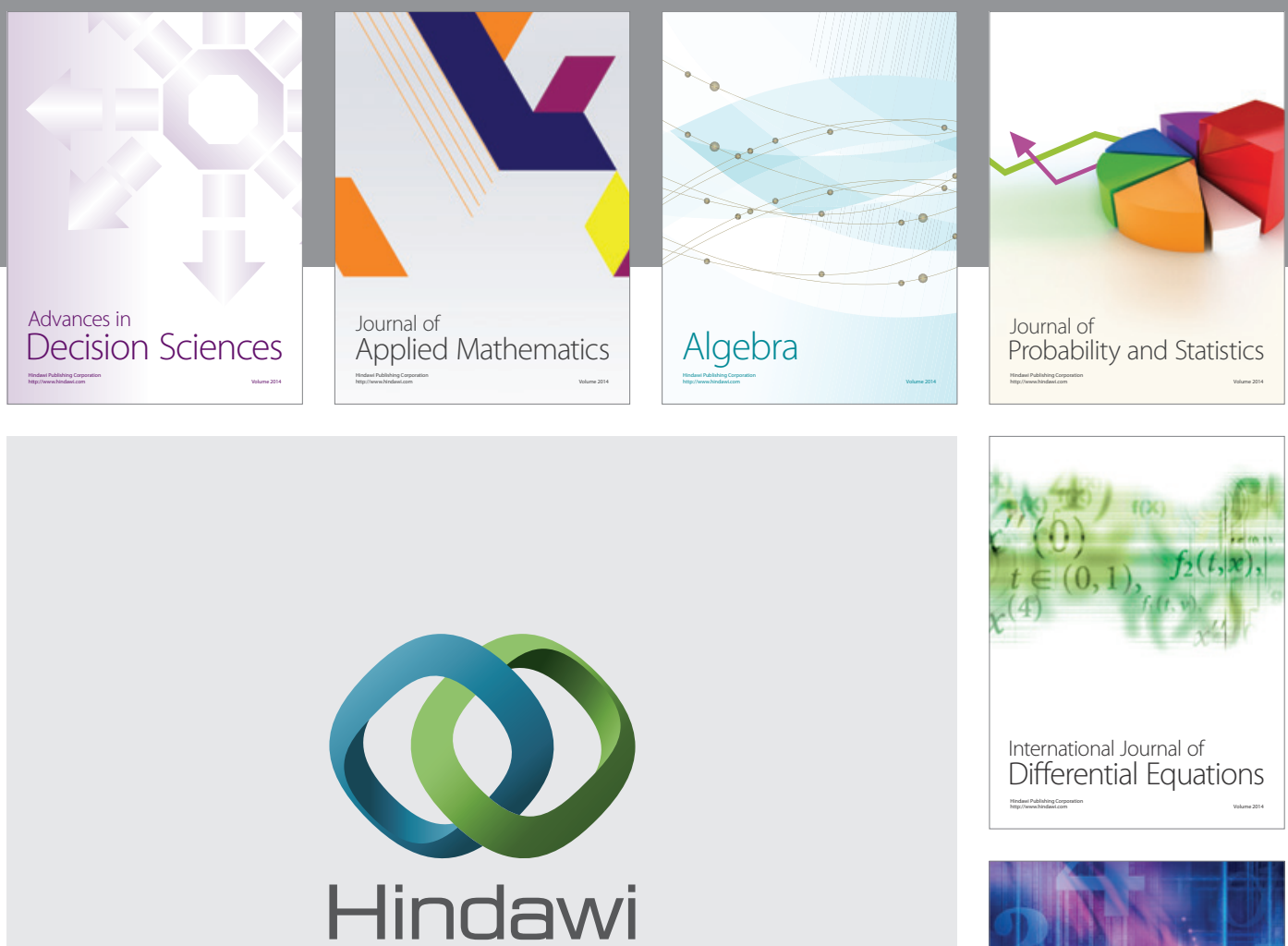

Submit your manuscripts at http://www.hindawi.com
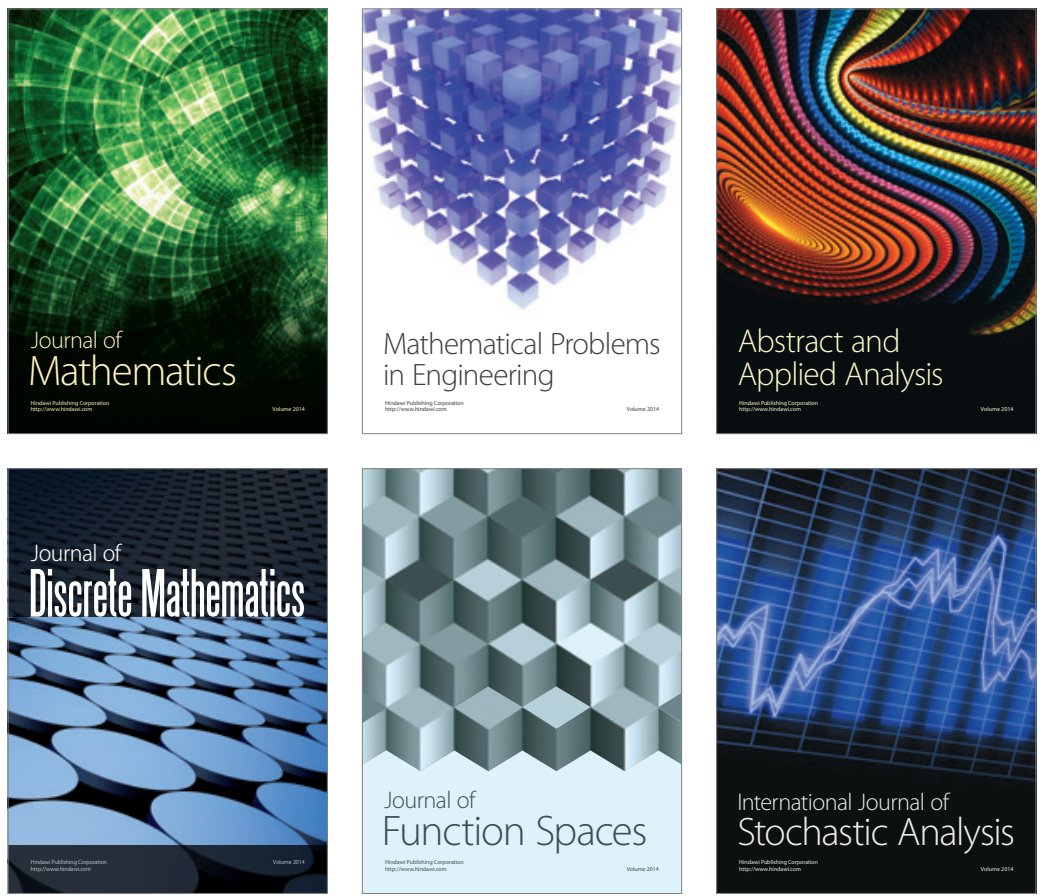

Journal of

Function Spaces

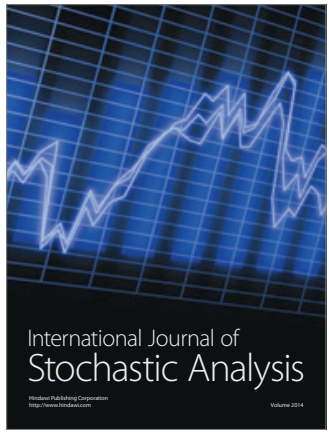

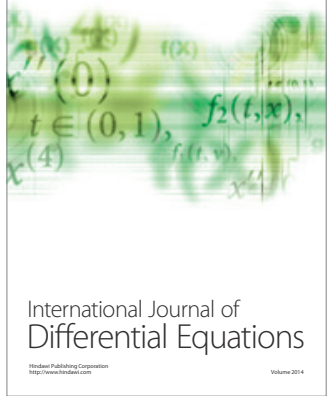
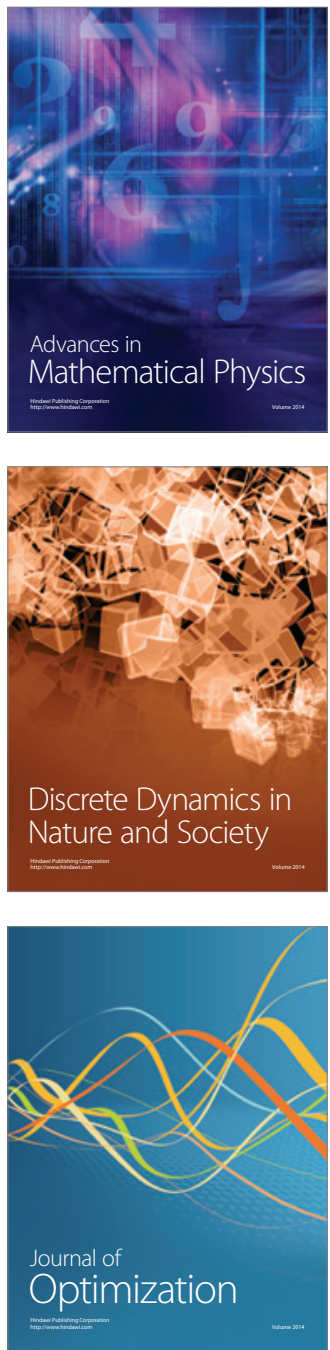